\title{
Degree-degree distribution in a power law random intersection graph with clustering
}

\author{
Mindaugas Bloznelis \\ Faculty of mathematics and informatics, \\ Vilnius university, 03225 Vilnius, Lithuania
}

\begin{abstract}
The bivariate distribution of degrees of adjacent vertices, degree-degree distribution, is an important network characteristic defining the statistical dependencies between degrees of adjacent vertices. We show the asymptotic degree-degree distribution of a sparse inhomogeneous random intersection graph and discuss its relation to the clustering and power law properties of the graph.
\end{abstract}

key words: degree-degree distribution, power law, clustering coefficient, random intersection graph, affiliation network.

\section{Introduction}

Correlations between degrees of adjacent vertices influence many network properties including the component structure, epidemic spreading, random walk performance, network robustness, etc., see [2], 8], [12, [18, [19] and references therein. The correlations are defined by the degree-degree distribution, i.e., the bivariate distribution of degrees of endpoints of a randomly chosen edge. In this paper we present an analytic study of the degree-degree distribution in a mathematically tractable random graph model of an affiliation network possessing tunable power law degree distribution and clustering coefficient. Our study is motivated by the interest in tracing the relation between the degree-degree distribution and clustering properties in a power law network.

Affiliation network and random intersection graph. An affiliation network defines adjacency relations between actors by using an auxiliary set of attributes. Let $V$ denote the set of actors (nodes of the network) and $W$ denote the auxiliary set of attributes. Every actor $v \in V$ is prescribed a collection $S_{v} \subset W$ of attributes and two actors $u, v \in V$ are declared adjacent in the network if they share some common attributes. For example one may interpret elements of $W$ as weights and declare two actors adjacent whenever the total weight of shared attributes is above some threshold value. Here we consider the simplest case, where $u, v \in V$ are called adjacent whenever they share at least one common attribute, i.e., $S_{u} \cap S_{v} \neq \emptyset$. Two popular examples of real affiliation networks are the film actor network, where two actors are declared adjacent if they have played in the same movie, and the collaboration network, where two scientists are declared adjacent if they have coauthored a publication. 
A plausible model of a large affiliation network is obtained by prescribing the collections of attributes to actors at random. In order to model the heterogeneity of human activity, every actor $v_{j} \in V$ is prescribed a random weight $y_{j}$ reflecting their activity. Similarly, a random weight $x_{i}$ is prescribed to each attribute $w_{i} \in W$ to model its attractiveness. Now an attribute $w_{i} \in W$ is included in the collection $S_{v_{j}}$ at random and with probability proportional to the attractiveness $x_{i}$ and activity $y_{j}$ (cf., [14], [17]). In this way we obtain a random graph on the vertex set $V$ sometimes called the inhomogeneous random intersection graph, see [5] and references therein. Before giving a detailed definition of this random graph model we mention a recent publication [16], which argues convincingly that in some social networks the 'heavy-tailed degree distribution is causally determined by similarly skewed distribution of human activity'. The empirical evidence reported in [16] suggests that the inhomogeneous random intersection graph can be considered as a realistic model of a power law affiliation network.

Rigorous model. Let $X_{1}, \ldots, X_{m}, Y_{1}, \ldots, Y_{n}$ be independent non-negative random variables such that each $X_{i}$ has the probability distribution $P_{1}$ and each $Y_{j}$ has the probability distribution $P_{2}$. Given realized values $X=\left\{X_{i}\right\}_{i=1}^{m}$ and $Y=\left\{Y_{j}\right\}_{j=1}^{n}$ we define the random bipartite graph $H_{X, Y}$ with the bipartition $V \cup W$, where $V=\left\{v_{1}, \ldots, v_{n}\right\}$ and $W=\left\{w_{1}, \ldots, w_{m}\right\}$. Every pair $\left\{w_{i}, v_{j}\right\}$ is linked in $H_{X, Y}$ with probability

$$
p_{i j}=\min \left\{1, \lambda_{i j}\right\}, \quad \text { where } \quad \lambda_{i j}=\frac{X_{i} Y_{j}}{\sqrt{n m}},
$$

independently of the other pairs $\{w, v\} \in W \times V$. For large $n$ and $m$, we typically have $\lambda_{i j}<1$ so that the probability $p_{i j}=\lambda_{i j}$ is proportional to the "activity" $Y_{j}$ of $v_{j}$ and the "attractiveness" $X_{i}$ of $w_{i}$. The inhomogeneous random intersection graph $G=G\left(P_{1}, P_{2}, n, m\right)$ defines the adjacency relation on the vertex set $V$ : vertices $u, v \in V$ are declared adjacent (denoted $u \sim v$ ) whenever $u$ and $v$ have a common neighbor in $H_{X, Y}$. We call this neighbor a witness of the adjacency relation $u \sim v$.

The random graph $G$ has several features that make it a convenient theoretical model of a real complex network. Firstly, the statistical dependence of neighboring adjacency relations in $G$ mimics that of real affiliation networks. In particular, $G$ admits a tunable clustering coefficient: For $m / n \rightarrow \beta \in(0,+\infty)$ as $m, n \rightarrow+\infty$, we have, see [7],

$$
\mathbf{P}\left(v_{1} \sim v_{2} \mid v_{1} \sim v_{3}, v_{2} \sim v_{3}\right)=\frac{\kappa}{\kappa+\sqrt{\beta}}+o(1) .
$$

Here $\kappa:=b_{1} b_{2}^{-1} a_{3} a_{2}^{-2}$ and $a_{i}=\mathbf{E} X_{1}^{i}, b_{j}=\mathbf{E} Y_{1}^{j}$. Secondly, an important feature of the model is its ability to produce a rich class of (asymptotic) degree distributions including power laws. Let $d(v)$ denote the degree of a vertex $v \in V$ in $G$. We note that, by the symmetry, random variables $d\left(v_{1}\right), \ldots, d\left(v_{n}\right)$ have the same probability distribution. The following result of [4] describes the asymptotic distribution of $d\left(v_{1}\right)$ as $n, m \rightarrow+\infty$.

Theorem 1. Let $m, n \rightarrow \infty$.

(i) Assume that $m / n \rightarrow \beta$ for some $\beta \in(0,+\infty)$. Suppose that $\mathbf{E} X_{1}^{2}<\infty$ and $\mathbf{E} Y_{1}<\infty$. Then $d\left(v_{1}\right)$ converges in distribution to the random variable

$$
d_{*}=\sum_{j=1}^{\Lambda_{1}} \tau_{j},
$$

where $\tau_{1}, \tau_{2}, \ldots$ are independent and identically distributed random variables independent of the random variable $\Lambda_{1}$. They are distributed as follows. For $r=0,1,2, \ldots$, we have

$$
\mathbf{P}\left(\tau_{1}=r\right)=\frac{r+1}{\mathbf{E} \Lambda_{0}} \mathbf{P}\left(\Lambda_{0}=r+1\right) \quad \text { and } \quad \mathbf{P}\left(\Lambda_{i}=r\right)=\mathbf{E} e^{-\lambda_{i}} \frac{\lambda_{i}^{r}}{r !}, \quad i=0,1 .
$$


Here $\lambda_{0}=X_{1} b_{1} \beta^{-1 / 2}$ and $\lambda_{1}=Y_{1} a_{1} \beta^{1 / 2}$.

(ii) Assume that $m / n \rightarrow+\infty$. Suppose that $\mathbf{E} X_{1}^{2}<\infty$ and $\mathbf{E} Y_{1}<\infty$. Then $d\left(v_{1}\right)$ converges in distribution to a random variable $\Lambda_{3}$ having the probability distribution

$$
\mathbf{P}\left(\Lambda_{3}=r\right)=\mathbf{E} e^{-\lambda_{3}} \frac{\lambda_{3}^{r}}{r !}, \quad r=0,1, \ldots
$$

Here $\lambda_{3}=Y_{1} a_{2} b_{1}$.

(iii) Assume that $m / n \rightarrow 0$. Suppose that that $\mathbf{E} X_{1}<\infty$. Then $\mathbf{P}\left(d\left(v_{1}\right)=0\right)=1-o(1)$.

We briefly explain the origin of $\Lambda_{i}, i=0,1,3$. The random variables $\Lambda_{0}$ and $\Lambda_{1}$ are limits (in distribution) of the degrees in the bipartite graph $H_{X, Y}$ of $w_{1}$ and $v_{1}$ respectively. That is, the number of attributes linked to $v_{1}$ converges in distribution to $\Lambda_{1}$ and the number of vertices linked to $w_{1}$ converges in distribution to $\Lambda_{0}$. Furthermore, the size-biased random variable $\tau_{j}$ counts the neighbors in $G$ of $v_{1}$ witnessed by an attribute $w_{j}$, given the event that $w_{j}$ is linked to $v_{1}$. As for $\Lambda_{3}$ it refers to the case where $m / n \rightarrow+\infty$. Here the number of attributes linked to $v_{1}$ grows to infinity (at the rate $\Theta(\sqrt{m / n})$ ), while the number of vertices linked to any given attribute vanishes (an attribute produces a single link with a small probability of order $\Theta(\sqrt{n / m})$ or has no link at all). Thus among many attributes linked to $v_{1}$ only a few contribute to the degree $d\left(v_{1}\right)$ by witnessing a single neighbor each. The number of neighbors converges in distribution to a mixed Poisson random variable $\Lambda_{3}$.

Using the fact that a Poisson random variable is highly concentrated around its mean one can show that for a power law distribution $\mathbf{P}\left(\lambda_{i}>r\right) \approx c_{i} r^{-\varkappa_{i}}$, with some $c_{i}, \varkappa_{i}>0$, we have $\mathbf{P}\left(\Lambda_{i}>r\right) \approx c_{i} r^{-\varkappa_{i}}$, for $i=0,1,3$, see Lemma 4. Here and below for real sequences $\left\{t_{r}\right\}_{r \geq 1}$ and $\left\{s_{r}\right\}_{r \geq 1}$ we denote $t_{r} \approx s_{r}$ whenever $t_{r} / s_{r} \rightarrow 1$ as $r \rightarrow+\infty$. Furthermore, the tail $\mathbf{P}\left(d^{*}>r\right)$ of a randomly stopped sum $d_{*}$ is as heavy as the heavier one of $Y_{1}$ and $\tau_{1}$, see, e.g., [1]. Hence, choosing a power law weights $X$ and $Y$ we obtain a power law asymptotic degree distributions, namely, the distributions of $d^{*}$ and $\Lambda_{3}$.

In what follows we will focus on local probabilities. Given $c>0$ and $\varkappa>1$, we say that a non-negative random variable $Z$ has the power law property $\mathcal{P}_{c, \varkappa}$ (denoted $Z \in \mathcal{P}_{c, \varkappa}$ ) if either $Z$ is integer valued and satisfies $\mathbf{P}(Z=r) \approx c r^{-\varkappa}$, or $Z$ is absolutely continuous with density $f_{Z}$ satisfying $f_{Z}(t)=(c+o(1)) t^{-\varkappa}$ as $t \rightarrow+\infty$.

Remark 1. Let $c, x>0$. Let $r \rightarrow+\infty$.

(i) Let $a>0$ and $\varkappa>3$. Assume that $\mathbf{E} e^{a Y_{1}}<\infty$ and $X_{1} \in \mathcal{P}_{c, \varkappa}$. Then $\mathbf{P}\left(d_{*}=r\right) \approx$ $c b_{1}^{\varkappa-1} \beta^{(3-\varkappa) / 2} r^{1-\varkappa}$.

(ii) Let $\varkappa>2$. Assume that $Y_{1} \in \mathcal{P}_{c, \varkappa}$ and $\mathbf{P}\left(X_{1}=x\right)=1$. Then $\mathbf{P}\left(d_{*}=r\right) \approx c\left(x^{2} b_{1}\right)^{\varkappa-1} r^{-\varkappa}$.

To show (i) we exploit the power law properties of the local probabilities of randomly stopped sums, like $d_{*}$, in the case where the summands are heavy tailed and the number of summands has a light tail (see, e.g., Theorem 4.30 of [9]). Unfortunately we are not aware of rigorous results establishing power law properties of the local probabilities of randomly stopped sums, like $d_{*}$, in the case where the number of summands is heavy tailed.

Degree-degree distribution. We are interested in the bivariate distribution of degrees of adjacent vertices. Denote $d_{1}=d\left(v_{1}\right), d_{2}=d\left(v_{2}\right)$ and let

$$
p\left(k_{1}, k_{2}\right)=\mathbf{P}\left(d_{1}=k_{1}+1, d_{2}=k_{2}+1 \mid v_{1} \sim v_{2}\right), \quad k_{1}, k_{2}=0,1, \ldots,
$$

denote the probabilities defining the conditional bivariate distribution of the ordered pair $\left(d_{1}, d_{2}\right)$, given the event that vertices $v_{1}$ and $v_{2}$ are adjacent. Let $\left(u^{*}, v^{*}\right)$ be an ordered pair of distinct 
vertices chosen uniformly at random from $V$. For the probability distribution of graph $G$ is invariant under permutations of its vertices, we have

$$
p\left(k_{1}, k_{2}\right)=p\left(k_{2}, k_{1}\right)=\mathbf{P}\left(d\left(u^{*}\right)=k_{1}+1, d\left(v^{*}\right)=k_{2}+1 \mid u^{*} \sim v^{*}\right) .
$$

In Theorem 2 below we show a first order asymptotics of $p\left(k_{1}, k_{2}\right)$ as $n, m \rightarrow+\infty$. Before formulating the theorem we introduce some notation. We remark that $d_{*}$ defined by $(2)$ depends on $Y_{1}$. By conditioning on the event $\left\{Y_{1}=y\right\}$ we obtain another random variable, denoted $d_{y}^{*}$, which has the compound Poisson distribution

$$
\mathbf{P}\left(d_{y}^{*}=r\right)=\mathbf{P}\left(d_{*}=r \mid Y_{1}=y\right)=\mathbf{P}\left(\sum_{j=1}^{N} \tau_{j}=r\right), \quad r=0,1, \ldots
$$

Here $N=N_{y}$ denotes a Poisson random variable which is independent of the iid sequence $\left\{\tau_{j}\right\}_{j \geq 1}$ and has mean $\mathbf{E} N_{y}=y a_{1} \beta^{1 / 2}, y \geq 0$. Given integers $k_{1}, k_{2}, r \geq 0$, denote

$$
\begin{aligned}
& q_{r}=\mathbf{E}\left(Y_{1} \mathbf{P}\left(d_{Y_{1}}^{*}=r \mid Y_{1}\right)\right)=\mathbf{E}\left(Y_{1} \mathbf{P}\left(d_{*}=r \mid Y_{1}\right)\right), \\
& p_{\beta}\left(k_{1}, k_{2}\right)=\frac{\beta}{b_{1}^{4} a_{2}} \sum_{r=0}^{k_{1} \wedge k_{2}}(r+1)(r+2) \mathbf{P}\left(\Lambda_{0}=r+2\right) q_{k_{1}-r} q_{k_{2}-r}, \\
& \tilde{p}(r)=r \mathbf{P}\left(\Lambda_{3}=r\right)\left(\mathbf{E} \Lambda_{3}\right)^{-1}, \quad p_{\infty}\left(k_{1}, k_{2}\right)=\tilde{p}\left(k_{1}+1\right) \tilde{p}\left(k_{2}+1\right) .
\end{aligned}
$$

Our main result is the following theorem.

Theorem 2. Let $m, n \rightarrow \infty$. Suppose that $\mathbf{E} X_{1}^{2}<\infty$ and $\mathbf{E} Y_{1}<\infty$.

(i) Assume that $m / n \rightarrow \beta$ for some $\beta \in(0,+\infty)$. Then for every $k_{1}, k_{2} \geq 0$ we have

$$
p\left(k_{1}, k_{2}\right)=p_{\beta}\left(k_{1}, k_{2}\right)+o(1),
$$

(ii) Assume that $m / n \rightarrow+\infty$. Then for every $k_{1}, k_{2} \geq 0$ we have

$$
p\left(k_{1}, k_{2}\right)=p_{\infty}\left(k_{1}, k_{2}\right)+o(1) .
$$

We note that the moment conditions $\mathbf{E} X_{1}^{2}<\infty$ and $\mathbf{E} Y_{1}<\infty$ of Theorem 2 are the minimal ones as the numbers $a_{2}=\mathbf{E} X_{1}^{2}$ and $b_{1}=\mathbf{E} Y_{1}$ enter (implicitly) both formulas (7) and (8).

Remark 2. In the case where $m / n \rightarrow+\infty$, the size biased probability distribution $\{\tilde{p}(r)\}_{r \geq 1}$ is the limiting distribution of $d\left(v_{1}\right)$ conditioned on the event $v_{1} \sim v_{2}$, i.e.,

$$
\mathbf{P}\left(d\left(v_{1}\right)=r \mid v_{1} \sim v_{2}\right)=\tilde{p}(r)+o(1), \quad r=1,2, \ldots
$$

Let us examine how the clustering property (presence of non-vanishing clustering coefficient) affects the structure of the asymptotic degree-degree distribution. Firstly we consider the case $m / n \rightarrow+\infty$, where the clustering coefficient vanishes (cf. (1)). In this case one may expect that the statistical dependence between neighboring adjacency relations fades away as $m, n \rightarrow+\infty$ and that the degrees of adjacent vertices are asymptotically independent. This is indeed the case as (8) and (9) imply that

$$
p\left(k_{1}, k_{2}\right)=\mathbf{P}\left(d\left(v_{1}\right)=k_{1}+1, \mid v_{1} \sim v_{2}\right) \mathbf{P}\left(d\left(v_{2}\right)=k_{2}+1 \mid v_{1} \sim v_{2}\right)+o(1) .
$$

For $m / n \rightarrow \beta \in(0,+\infty)$ the random graph $G$ admits a non-vanishing clustering coefficient. Now, the statistical dependence of neighboring adjacency relations persists as $n, m \rightarrow+\infty$, and 
the asymptotic bivariate degree-degree distribution is not a product of marginal asymptotic degree distributions, see (7). One can show that in this case the degrees of adjacent vertices are positively correlated and $G$ admits a non-vanishing positive Newman's assortativity coefficient, provided that the vertex degree distribution has a finite third moment (cf. [6]).

Several examples of power law degree-degree distributions are considered in Theorem 3 below.

Theorem 3. Let $a, \beta, c, x>0$.

(i) Assume that $\mathbf{E} X_{1}^{2}<\infty$ and $Y_{1} \in \mathcal{P}_{c, \varkappa}$, for some $\varkappa>2$. Then for $k_{1}, k_{2} \rightarrow+\infty$ we have

$$
p_{\infty}\left(k_{1}, k_{2}\right)=(1+o(1)) c^{2} a_{2}^{2 \varkappa-4} b_{1}^{2 \varkappa-6}\left(k_{1} k_{2}\right)^{1-\varkappa} .
$$

(ii) Assume that $\mathbf{E} e^{a Y_{1}}<\infty$ and $X_{1} \in \mathcal{P}_{c, \varkappa}$, for some $\varkappa>3$. Let $k_{1}, k_{2} \rightarrow+\infty$ so that $k_{1} \leq k_{2}$. Suppose that either $k_{2}-k_{1} \rightarrow+\infty$ or $k_{2}-k_{1}=k$, for an arbitrary, but fixed integer $k \geq 0$. Then

$$
p_{\beta}\left(k_{1}, k_{2}\right)=(1+o(1)) \frac{\beta}{b_{1}^{4} a_{2}} \times\left\{\begin{array}{lll}
b_{1} c_{1}^{*} c_{2}^{*} k_{1}^{2-\varkappa}\left(k_{2}-k_{1}\right)^{1-\varkappa} & \text { if } k_{2}-k_{1} \rightarrow+\infty, \\
c_{1}^{*} c_{3, k}^{*} k_{1}^{2-\varkappa} & \text { if } k_{2}-k_{1}=k .
\end{array}\right.
$$

Here $c_{1}^{*}=c\left(b_{1} \beta^{-1 / 2}\right)^{\varkappa-1}, c_{2}^{*}=c b_{1}^{\varkappa-2} b_{2} \beta^{(3-\varkappa) / 2}$, and $c_{3, k}^{*}=\sum_{i \geq 0} q_{i} q_{k+i}$. Furthermore, we have $q_{r} \approx c_{2}^{*} r^{1-\varkappa}$. We recall that $q_{i}$ is defined in (6).

(iii) Assume that $\mathbf{P}\left(X_{1}=x\right)=1$ and $Y_{1} \in \mathcal{P}_{c, \varkappa}$, for some $\varkappa>2$. For $k_{1}, k_{2} \rightarrow+\infty$ we have

$$
p_{\beta}\left(k_{1}, k_{2}\right)=(1+o(1)) c^{2} x^{4 \varkappa-8} b_{1}^{2 \varkappa-6}\left(k_{1} k_{2}\right)^{1-\varkappa} .
$$

We remark, that example (iii) refers to the clustering regime $(m / n \rightarrow \beta \in(0,+\infty))$, where neighboring adjacency relations are statistically dependent. Hence $p_{\beta}(\cdot, \cdot)$ is not a product of marginal probabilities. An interesting fact is that for $k_{1}, k_{2} \rightarrow+\infty$ the tail of $p_{\beta}\left(k_{1}, k_{2}\right)$ is asymptotically a product of independent marginals. Here we observe a situation, where heavy tailed weights of actors $Y_{j}$ define the power law tails of $p_{\beta}(\cdot, \cdot)$ and outperform the light weights of attributes $X_{i}$.

Our final remark is about the case where $m / n \rightarrow 0$. By Theorem 1 , in this case the edges of a sparse inhomogeneous random intersection graph span a subgraph on $o(n)$ randomly selected vertices leaving the remaining $(1-o(1)) n$ vertices isolated. Consequently, the subgraph is relatively dense and we do not expect stochastically bounded degrees of endpoints of adjacent vertices.

Related work. The influence of degree-degree correlations on the network properties have been studied by many authors, see, e.g., [8], [12], 18], [19] and references therein. The asymptotic degree-degree distribution in a preferential attachment random graph with tunable power law degree distribution was shown in [11]. Our model and approach are much different. To our best knowledge the present paper is the first attempt to trace the relation between the degree-degree distribution and the clustering property in a power law network. Connections between Newman's assortativity coefficient and the clustering coefficient in related random graph models have been discussed in [6].

The present paper complements, revises and extends the results of [3], presented at the 12th Workshop on Algorithms and Models for the Web Graph, WAW 2015. In particular, the factor $b_{1}$ is included in (11). It was missing in the respective formula (7) of [3].

\section{Proof}

Here we prove Theorems 2, 3 and Remarks 1, 2, Before the proof we collect some notation. We will assume throughout the proof that $m=m(n) \rightarrow+\infty$ as $n \rightarrow+\infty$. The expressions 
$o(\cdot), O(\cdot)$ will always refer to the case where $n \rightarrow+\infty$. We use the notation $o_{P}(\cdot)$ and $O_{P}(\cdot)$ consistently with [13]. Given two real sequences $\left\{a_{n}\right\}_{n \geq 1}$ and $\left\{b_{n}\right\}_{n \geq 1}$ we write $a_{n} \simeq b_{n}$ to denote the fact that $\left(a_{n}-b_{n}\right) m n=o(1)$. By $\mathbb{I}_{\mathcal{A}}$ we denote the indicator function of an event $\mathcal{A}$ and $\overline{\mathbb{I}}_{\mathcal{A}}=1-\mathbb{I}_{\mathcal{A}}=\mathbb{I}_{\overline{\mathcal{A}}}$ denotes the indicator of the complement event $\overline{\mathcal{A}}$.

In the proof we often use the following facts: For a random variable $Z$ and a sequence of events $\left\{\mathcal{K}_{n}\right\}_{n \geq 1}$ defined on the same probability space we have that $\mathbf{E}|Z|<\infty$ and $\lim _{n} \mathbf{P}\left(\mathcal{K}_{n}\right)=0$ imply $\mathbf{E}\left(\mathbb{L}_{\mathcal{K}_{n}} Z\right)=o(1)$; for a sequence of random variables $\left\{Z_{n}\right\}_{n \geq 1}$ the conditions $\exists C>0$ such that $\left|Z_{n}\right| \leq C$ almost surely $\forall n$, and $Z_{n}=o_{P}(1)$ imply $\mathbf{E}\left|Z_{n}\right|=o(1)$.

We denote by $\mathbf{P}^{\star}$ and $\mathbf{E}^{\star}$ (respectively $\tilde{\mathbf{P}}$ and $\tilde{\mathbf{E}}$ ) the conditional probability and expectation given $X_{1}, Y_{1}, Y_{2}$ (respectively $X, Y$ ). For random variables $\xi, \zeta$ defined on the same probability space as $X, Y$ we denote by $d_{T V}^{\star}(\xi, \zeta)$ (respectively $\left.\tilde{d}_{T V}(\xi, \zeta)\right)$ the total variation distance between the conditional distributions of $\xi$ and $\zeta$ given $X_{1}, Y_{1}, Y_{2}$ (respectively $X, Y$ ). Given a sequence of random variables $\left\{Z_{n}\right\}_{n \geq 1}$, defined on the same probability space as $Y_{1}, Y_{2}, X_{1}$, the notation $Z_{n}=o_{P^{\star}}(1)$ means that, given any realized values $Y_{1}, Y_{2}, X_{1}$ and any $\varepsilon>0$, we have $\mathbf{P}^{\star}\left(Z_{n}>\right.$ $\varepsilon)=o(1)$. We note that for $\left\{Z_{n}\right\}_{n \geq 1}$ independent of $Y_{1}, Y_{2}, X_{1}$ we have $Z_{n}=o_{P^{\star}}(1) \Leftrightarrow Z_{n}=$ $o_{P}(1)$.

The degree of a vertex $v_{i} \in V$ is denoted $d_{i}=d\left(v_{i}\right)$. The number of common neighbors of $v_{i}, v_{j} \in V$ is denoted $d_{i j}$. For a vertex $v \in V$ and attribute $w \in W$ we denote by $\{w \rightarrow v\}$ the event that $v$ and $w$ are linked in $H$. Introduce the events

$$
\mathcal{A}_{i}=\left\{w_{i} \rightarrow v_{1}, w_{i} \rightarrow v_{2}\right\}, \quad 1 \leq i \leq m .
$$

We write for short

$$
\mathbb{I}_{i j}=\mathbb{I}_{\left\{w_{i} \rightarrow v_{j}\right\}}, \quad \overline{\mathbb{I}}_{i j}=1-\mathbb{I}_{i j}, \quad \mathbf{I}_{i j}=\mathbb{I}_{\left\{\lambda_{i j} \leq 1\right\}}, \quad \overline{\mathbf{I}}_{i j}=1-\mathbf{I}_{i j} .
$$

Let $\mathbf{L}=\left(L_{0}, L_{1}, L_{2}\right)$ denote the random vector with marginal random variables

$$
L_{0}=u_{1}, \quad L_{1}=\sum_{2 \leq i \leq m} \mathbb{I}_{i 1} u_{i}, \quad L_{2}=\sum_{2 \leq i \leq m} \mathbb{I}_{i 2} u_{i}, \quad u_{i}=\sum_{3 \leq j \leq n} \mathbb{I}_{i j}, \quad 1 \leq i \leq m .
$$

Let $\Lambda_{i}, 0 \leq i \leq 4$ denote random variables having mixed Poisson distributions

$$
\mathbf{P}\left(\Lambda_{i}=r\right)=\mathbf{E}\left(e^{-\lambda_{i}} \lambda_{i}^{r} / r !\right), \quad r=0,1,2, \ldots,
$$

where

$$
\lambda_{0}=X_{1} b_{1} \beta^{-1 / 2}, \quad \lambda_{1}=Y_{1} a_{1} \beta^{1 / 2}, \quad \lambda_{2}=Y_{2} a_{1} \beta^{1 / 2}, \quad \lambda_{3}=Y_{1} a_{2} b_{1}, \quad \lambda_{4}=Y_{2} a_{2} b_{1}
$$

are random variables. We assume that conditionally, given $Y_{1}, Y_{2}, X_{1}$, the random variables $\Lambda_{i}$, $0 \leq i \leq 4$ are independent. Define random variables $d_{Y_{1}}^{*}=\sum_{i=1}^{\Lambda_{1}} \tau_{i}$ and $d_{Y_{2}}^{*}=\sum_{i=1}^{\Lambda_{2}} \tau_{i}^{\prime}$. Here $\tau_{i}, \tau_{i}^{\prime}, i \geq 1$ are independent and identically distributed random variables, which are independent of $Y_{1}, Y_{2}, X_{1}$ and have distribution (3). Define the events

$$
\begin{array}{ll}
\mathcal{U}_{k_{1}, k_{2}}=\left\{d_{1}=k_{1}+1, d_{2}=k_{2}+1\right\}, & \mathcal{U}_{r, r_{1}, r_{2}}^{*}=\left\{\mathbf{L}=\left(r, r_{1}, r_{2}\right)\right\}, \\
\mathcal{U}_{r, r_{1}, r_{2}}^{* *}=\left\{\Lambda_{0}=r, d_{Y_{1}}^{*}=r_{1}, d_{Y_{2}}^{*}=r_{2}\right\}, & \mathcal{U}_{r_{1}, r_{2}}^{* * *}=\left\{\Lambda_{3}=r_{1}, \Lambda_{4}=r_{2}\right\} .
\end{array}
$$

Define random variables $\hat{a}_{k}=m^{-1} \sum_{2 \leq i \leq m} X_{i}^{k}$ and $\hat{b}_{k}=n^{-1} \sum_{3 \leq j \leq n} Y_{j}^{k}$.

Proof of Theorem 2. Proof of (i). The intuition behind formula (7) is that with a high probability the adjacency relation $v_{1} \sim v_{2}$ as well as all common neighbors of $v_{1}$ and $v_{2}$ are witnessed, by 
the same common attribute (all attributes having equal chances). Furthermore, conditionally on the event that this attribute is $w_{1}$, and given $Y_{1}, Y_{2}, X_{1}$, we have that the random variables $d_{12}, d_{1}-1-d_{12}=: d_{1}^{\prime}, d_{2}-1-d_{12}=: d_{2}^{\prime}$ are asymptotically independent. We note that $d_{1}^{\prime}$ and $d_{2}^{\prime}$ count individual (not shared) neighbors of $v_{1}$ and $v_{2}$. The individual neighbors of $v_{1}$ (of $v_{2}$ ) are attracted by all attributes linked to $v_{1}$ (to $v_{2}$ ), but $w_{1}$, while the common neighbors are attracted by the attribute $w_{1}$. The (conditional given $Y_{1}, Y_{2}, X_{1}$ ) asymptotic independence of $d_{12}, d_{1}^{\prime}, d_{2}^{\prime}$ comes from the fact that these random variables are mainly related to each other via average characteristics $\hat{a}_{1}, \hat{b}_{1}$ which are asymptotically constant, by the law of large numbers. Now, using Theorem 1 we identify limiting distributions of $d_{1}^{\prime}, d_{2}^{\prime}$. Finally, the limiting distribution for the number of vertices from $\left\{v_{3}, \ldots, v_{n}\right\}$ attracted by $w_{1}$ is that of $\Lambda_{0}$.

We briefly outline the proof. In the first step we show that

$$
p\left(k_{1}, k_{2}\right)=\frac{\mathbf{P}\left(\mathcal{U}_{k_{1}, k_{2}} \cap\left\{v_{1} \sim v_{2}\right\}\right)}{\mathbf{P}\left(v_{1} \sim v_{2}\right)}=\frac{\mathbf{P}\left(\mathcal{U}_{k_{1}, k_{2}} \cap\left(\cup_{i} \mathcal{A}_{i}\right)\right)}{\left.\mathbf{P}\left(\cup_{i} \mathcal{A}_{i}\right)\right)}=\frac{n m}{a_{2} b_{1}^{2}} \mathbf{P}\left(\mathcal{U}_{k_{1}, k_{2}} \cap \mathcal{A}_{1}\right)+o(1) .
$$

Then using the total probability formula we split

$$
\mathbf{P}\left(\mathcal{U}_{k_{1}, k_{2}} \cap \mathcal{A}_{1}\right)=\sum_{r=0}^{k_{1} \wedge k_{2}} \mathbf{P}\left(\mathcal{U}_{k_{1}, k_{2}} \cap\left\{d_{12}=r\right\} \cap \mathcal{A}_{1}\right) .
$$

In the second step we show that for every $r=0,1, \ldots, k_{1} \wedge k_{2}$

$$
\mathbf{P}\left(\mathcal{U}_{k_{1}, k_{2}} \cap\left\{d_{12}=r\right\} \cap \mathcal{A}_{1}\right) \simeq \mathbf{P}\left(\mathcal{U}_{r, k_{1}-r, k_{2}-r}^{*} \cap \mathcal{A}_{1}\right) .
$$

In the final step we show that for $r, r_{1}, r_{2}=0,1,2 \ldots$

$$
\mathbf{P}\left(\mathcal{U}_{r, r_{1}, r_{2}}^{*} \cap \mathcal{A}_{1}\right) \simeq \mathbf{E}\left(\lambda_{11} \lambda_{12} \mathbf{P}^{\star}\left(\mathcal{U}_{r, r_{1}, r_{2}}^{* *}\right)\right)
$$

Now the simple identity

$$
n m \mathbf{E}\left(\lambda_{11} \lambda_{12} \mathbf{P}^{\star}\left(\mathcal{U}_{r, r_{1}, r_{2}}^{* *}\right)\right)=\frac{\beta}{b_{1}^{2}}(r+1)(r+2) \mathbf{P}\left(\Lambda_{0}=r+2\right) q_{r_{1}} q_{r_{2}}
$$

completes the proof of (7). Finally, we remark that in order to prove the result under the minimal moment conditions $\mathbf{E} Y_{1}<\infty$ and $\mathbf{E} X_{1}^{2}<\infty$, we invoke, when necessary, a truncation argument, which makes our presentation some more involved.

Step 1. Here we prove (14). To this aim we show that

$$
\begin{aligned}
& \mathbf{P}\left(\mathcal{U}_{k_{1}, k_{2}} \cap\left\{v_{1} \sim v_{2}\right\}\right)=m \mathbf{P}\left(\mathcal{U}_{k_{1}, k_{2}} \cap \mathcal{A}_{1}\right)+o\left(n^{-1}\right), \\
& \mathbf{P}\left(\left\{v_{1} \sim v_{2}\right\}\right)=n^{-1} a_{2} b_{1}^{2}+o\left(n^{-1}\right) .
\end{aligned}
$$

Since $\mathbf{P}\left(\mathcal{A}_{i}\right) \leq \mathbf{E} \lambda_{i 1} \lambda_{i 2}=a_{2} b_{1}^{2}(n m)^{-1}$, relations (18) imply (14). We only prove the second relation. The proof of the first one is much the same. Fix a small $0<\delta<1$ and introduce truncation events

$$
\mathcal{H}_{i}=\left\{Y_{i}<\delta \sqrt{n}\right\}, \quad i=1,2 .
$$

We have

$$
\begin{aligned}
\mathbf{P}\left(\left\{v_{1} \sim v_{2}\right\}\right)= & \mathbf{P}\left(\left\{v_{1} \sim v_{2}\right\} \cap \mathcal{H}_{1} \cap \mathcal{H}_{2}\right)+\mathbf{P}\left(\left\{v_{1} \sim v_{2}\right\} \cap \overline{\mathcal{H}}_{1} \cap \mathcal{H}_{2}\right) \\
& +\mathbf{P}\left(\left\{v_{1} \sim v_{2}\right\} \cap \mathcal{H}_{1} \cap \overline{\mathcal{H}}_{2}\right)+\mathbf{P}\left(\left\{v_{1} \sim v_{2}\right\} \cap \overline{\mathcal{H}}_{1} \cap \overline{\mathcal{H}}_{2}\right) \\
=: & p_{1}^{\prime}+p_{2}^{\prime}+p_{3}^{\prime}+p_{4}^{\prime} .
\end{aligned}
$$


Now we evaluate $p_{1}^{\prime}$ and construct upper bounds for $p_{i}^{\prime}, i=2,3,4$. Using the independence of $Y_{1}, Y_{2}$ and Markov's inequality we obtain

$$
p_{4}^{\prime} \leq \mathbf{P}\left(\overline{\mathcal{H}}_{1} \cap \overline{\mathcal{H}}_{2}\right) \leq \delta^{-2} n^{-1}\left(\mathbf{E}\left(Y_{1} \overline{\mathbb{I}}_{\mathcal{H}_{1}}\right)\right)^{2} .
$$

Next, using the identity $\left\{v_{1} \sim v_{2}\right\}=\cup_{1 \leq i \leq m} \mathcal{A}_{i}$ and inequality

$$
\mathbf{P}\left(\mathcal{A}_{1} \cap \overline{\mathcal{H}}_{1}\right)=\mathbf{E}\left(\mathbf{P}^{\star}\left(\mathcal{A}_{1}\right) \mathbb{I}_{\overline{\mathcal{H}}_{1}}\right) \leq \mathbf{E}\left(\lambda_{11} \lambda_{21} \overline{\mathcal{H}}_{1}\right)
$$

we obtain

$$
p_{2}^{\prime} \leq \sum_{1 \leq i \leq m} \mathbf{P}\left(\mathcal{A}_{i} \cap \overline{\mathcal{H}}_{1}\right)=m \mathbf{P}\left(\mathcal{A}_{1} \cap \overline{\mathcal{H}}_{1}\right) \leq \frac{a_{2} b_{1}}{n} \mathbf{E} Y_{1} \overline{\mathbb{I}}_{\mathcal{H}_{1}}
$$

Clearly, 21 extends to $p_{3}^{\prime}$. An upper bound on $p_{1}^{\prime}$ is obtained in a similar way,

$$
p_{1}^{\prime} \leq \mathbf{P}\left(\left\{v_{1} \sim v_{2}\right\}\right) \leq \sum_{1 \leq i \leq m} \mathbf{P}\left(\mathcal{A}_{i}\right) \leq \sum_{1 \leq i \leq m} \mathbf{E} \lambda_{1 i} \lambda_{2 i}=n^{-1} a_{2} b_{1}^{2} .
$$

To get a lower bound we invoke inclusion-exclusion. We have

$$
p_{1}^{\prime} \geq \sum_{1 \leq i \leq m} \mathbf{P}\left(\mathcal{A}_{i} \cap \mathcal{H}_{1} \cap \mathcal{H}_{2}\right)-\sum_{1 \leq i<j \leq m} \mathbf{P}\left(\mathcal{A}_{i} \cap \mathcal{A}_{j} \cap \mathcal{H}_{1} \cap \mathcal{H}_{2}\right)=: m p^{\prime}-\left(\begin{array}{c}
m \\
2
\end{array}\right) p^{\prime \prime}
$$

where $p^{\prime}=\mathbf{P}\left(\mathcal{A}_{1} \cap \mathcal{H}_{1} \cap \mathcal{H}_{2}\right)$ and $p^{\prime \prime}=\mathbf{P}\left(\mathcal{A}_{1} \cap \mathcal{A}_{2} \cap \mathcal{H}_{1} \cap \mathcal{H}_{2}\right)$. In order to evaluate $p^{\prime}=$ $\mathbf{E}\left(\mathbf{P}^{\star}\left(\mathcal{A}_{1}\right) \mathbb{I}_{\mathcal{H}_{1} \cap \mathcal{H}_{2}}\right)$ we invoke the inequalities

$$
\left(1-\overline{\mathbf{I}}_{11}-\overline{\mathbf{I}}_{12}\right) \lambda_{11} \lambda_{12} \leq \mathbf{I}_{11} \mathbf{I}_{12} \lambda_{11} \lambda_{12}=\mathbf{I}_{11} \mathbf{I}_{12} \mathbf{P}^{\star}\left(\mathcal{A}_{1}\right) \leq \mathbf{P}^{\star}\left(\mathcal{A}_{1}\right) \leq \lambda_{11} \lambda_{12} .
$$

We obtain

$$
p^{\prime} \geq \mathbf{E}\left(\mathbf{P}^{\star}\left(\mathcal{A}_{1}\right) \mathbb{I}_{\mathcal{H}_{1} \cap \mathcal{H}_{2}} \mathbf{I}_{11} \mathbf{I}_{12}\right) \geq(n m)^{-1}\left(a_{2} b_{1}^{2}-R\right),
$$

where $R=\mathbf{E}\left(Y_{1} Y_{2} X_{1}^{2}\left(\overline{\mathbf{I}}_{11}+\overline{\mathbf{I}}_{12}+\overline{\mathbb{I}}_{\mathcal{H}_{1} \cap \mathcal{H}_{2}}\right)\right)$. The next upper bound for $p^{\prime \prime}$ is simple

$$
p^{\prime \prime}=\mathbf{E}\left(\mathbf{P}^{\star}\left(\mathcal{A}_{1}\right) \mathbf{P}^{\star}\left(\mathcal{A}_{2}\right) \mathbb{I}_{\mathcal{H}_{1} \cap \mathcal{H}_{2}}\right) \leq \mathbf{E}\left(\lambda_{11} \lambda_{12} \lambda_{21} \lambda_{22} \mathbb{I}_{\mathcal{H}_{1} \cap \mathcal{H}_{2}}\right) \leq \delta^{2} n^{-1} m^{-2} \mathbf{E}\left(Y_{1} Y_{2} X_{1}^{2} X_{2}^{2}\right) .
$$

Collecting (25), (26) in (23) we obtain a lower bound for $p_{1}^{\prime}$. Combining this lower bound with (22) we obtain

$$
n^{-1}\left(a_{2} b_{1}^{2}-R-\delta^{2} a_{2}^{2} b_{1}^{2}\right) \leq p_{1}^{\prime} \leq n^{-1} a_{2} b_{1}^{2} .
$$

Finally, we choose $\delta=\delta_{n}$ converging to 0 slowly enough so that $\delta \sqrt{n} \rightarrow+\infty$ and $\delta^{-1} \mathbf{E}\left(Y_{1} \overline{\mathbb{I}}_{H_{1}}\right) \rightarrow$ 0 . We obtain from (20), 21) that $p_{2}^{\prime}, p_{3}^{\prime}, p_{4}^{\prime}=o\left(n^{-1}\right)$ and $(27)$ implies that $p_{1}^{\prime}=n^{-1} a_{2} b_{1}^{2}+o\left(n^{-1}\right)$. Hence (18) follows from (19).

Step 2. Here we prove $(16)$. Let us note that event $\mathcal{A}_{1}$ implies that $L_{0}$ counts common neighbors of $v_{1}$ and $v_{2}$ witnessed by $w_{1}$. In the case where $L_{0} \neq d_{12}$ there should be a common neighbor of $v_{1}, v_{2}$ witnessed by some attribute $w_{i}$ other than $w_{1}$ or witnessed by two different attributes, say $w_{i_{1}}, w_{i_{2}} \in W \backslash\left\{w_{1}\right\}$. We introduce related events

$$
\begin{aligned}
& \mathcal{B}_{00}=\left\{\mathbb{I}_{i 1} \mathbb{I}_{i 2}=1 \text { for some } 2 \leq i \leq m\right\} \\
& \mathcal{B}_{01}=\left\{\mathbb{I}_{i_{1} 1} \mathbb{I}_{i_{1} j} \mathbb{I}_{i_{2} 2} \mathbb{I}_{i_{2} j}=1 \text { for some } 3 \leq j \leq n \text { and } 2 \leq i_{1} \neq i_{2} \leq m\right\}
\end{aligned}
$$

and observe that on the event $\mathcal{A}_{1} \cap \overline{\left(\mathcal{B}_{00} \cup \mathcal{B}_{01}\right)}$ we have $L_{0}=d_{12}$. Next, assuming that events $\mathcal{A}_{1}$ and $L_{0}=d_{12}$ hold we consider $L_{k}$ for $k=1,2$. The sum $L_{k}$ counts pairs $w_{i} \rightarrow v_{j}$, for $i \geq 1$, 
$j \geq 3$, such that $v_{j}$ is a neighbor of $v_{k}$ witnessed by $w_{i}$. Generally, we have $L_{k} \geq d_{k}-1-d_{12}$. This inequality is strict if some common neighbor of $v_{1}, v_{2}$ witnessed by $w_{1}$ is also witnessed as a neighbor of $v_{k}$ by some other attribute $w_{i}, i \geq 2$. The inequality is also strict in the case where some neighbor of $v_{k}$ is witnessed by two or more distinct attributes. If we rule out both of these possibilities, we have the equality $L_{k}=d_{k}-1-d_{12}$. We introduce the corresponding undesired events

$$
\begin{aligned}
& \mathcal{B}_{k 0}=\left\{\mathbb{I}_{i k} \mathbb{I}_{i j} \mathbb{I}_{1 j}=1 \text { for some } 3 \leq j \leq n \text { and } 2 \leq i \leq m\right\} \\
& \mathcal{B}_{k 1}=\left\{\mathbb{I}_{i_{1} k} \mathbb{I}_{i_{1} j} \mathbb{I}_{i_{2} k} \mathbb{I}_{i_{2} j}=1 \text { for some } 3 \leq j \leq n \text { and } 2 \leq i_{1}<i_{2} \leq m\right\}
\end{aligned}
$$

Finally, we conclude that if $\mathcal{A}_{1}$ holds and at least one of the following three relations fails

$$
L_{0}=d_{12}, \quad L_{1}+L_{0}=d_{1}-1, \quad L_{2}+L_{0}=d_{2}-1
$$

then at least one of the events $\mathcal{B}_{l}, \mathcal{B}_{l j}, 0 \leq l \leq 2,0 \leq j \leq 1$, occurs. Hence, we have

$$
\left|\mathbf{P}\left(\mathcal{U}_{k_{1}, k_{2}} \cap\left\{d_{12}=r\right\} \cap \mathcal{A}_{1}\right)-\mathbf{P}\left(\mathcal{U}_{r, k_{1}-r, k_{2}-r}^{*} \cap \mathcal{A}_{1}\right)\right| \leq \sum_{0 \leq l \leq 2} \sum_{0 \leq j \leq 1} \mathbf{P}\left(\mathcal{A}_{1} \cap \mathcal{B}_{l j}\right) .
$$

Next we prove that the quantity on the right is $o\left((n m)^{-1}\right)$. For this purpose we estimate

$$
\mathbf{P}\left(\mathcal{A}_{1} \cap \mathcal{B}_{l j}\right)=\mathbf{E}\left(\mathbf{P}^{\star}\left(\mathcal{A}_{1}\right) \mathbf{P}^{\star}\left(\mathcal{B}_{l j}\right)\right) \leq(m n)^{-1} \mathbf{E}\left(Y_{1} Y_{2} X_{1}^{2} \mathbf{P}^{\star}\left(\mathcal{B}_{l j}\right)\right)
$$

and show that for any realized values $Y_{1}, Y_{2}, X_{1}$ we have

$$
\mathbf{P}^{\star}\left(\mathcal{B}_{l j}\right)=o(1), \quad \text { for } \quad 0 \leq l \leq 2, \quad 0 \leq j \leq 1 .
$$

Since $\mathbf{P}^{*}(\cdot) \leq 1$ and $\mathbf{E} Y_{1} Y_{2} X_{1}^{2}<\infty$, Lebesgue's dominated convergence theorem then implies the desired bound $\mathbf{E}\left(Y_{1} Y_{2} X_{1}^{2} \mathbf{P}^{\star}\left(\mathcal{B}_{l j}\right)\right)=o(1)$.

Let us show 299 . For this purpose we write events $\mathcal{B}_{l j}$ in the form $\mathcal{B}_{l j}=\left\{S_{l j} \geq 1\right\}$, where

$$
\begin{aligned}
S_{00} & =\sum_{2 \leq i \leq m} \mathbb{I}_{i 1} \mathbb{I}_{i 2}, & S_{01} & =\sum_{3 \leq j \leq n} \sum_{2 \leq i_{1} \neq i_{2} \leq m} \mathbb{I}_{i_{1} 1} \mathbb{I}_{i_{1} j} \mathbb{I}_{i_{2} 2} \mathbb{I}_{i_{2} j}, \\
S_{k 0} & =\sum_{3 \leq j \leq n} \sum_{2 \leq i \leq m} \mathbb{I}_{i k} \mathbb{I}_{i j} \mathbb{I}_{1 j}, & S_{k 1} & =\sum_{3 \leq j \leq n} \sum_{2 \leq i_{1}<i_{2} \leq m} \mathbb{I}_{i_{1} k} \mathbb{I}_{i_{1} j} \mathbb{I}_{i_{2} k} \mathbb{I}_{i_{2} j},
\end{aligned}
$$

and apply the inequality

$$
\mathbf{P}^{\star}\left(S_{l j} \geq 1\right) \leq \varepsilon+\mathbf{P}^{\star}\left(\tilde{\mathbf{E}} S_{l j} \geq \varepsilon\right), \quad \forall \varepsilon>0 .
$$

Let us briefly explain (31). We apply the trivial inequality $Z \leq \varepsilon+Z \mathbb{I}_{\{Z>\varepsilon\}}$ to the random variable $Z=\tilde{\mathbf{P}}\left(S_{l j} \geq 1\right)$ and obtain

$$
\tilde{\mathbf{P}}\left(S_{l j} \geq 1\right) \leq \varepsilon+\mathbb{I}_{\left\{\tilde{\mathbf{P}}\left(S_{l j} \geq 1\right)>\varepsilon\right\}} \leq \varepsilon+\mathbb{I}_{\left\{\tilde{\mathbf{E}} S_{l j}>\varepsilon\right\}} .
$$

The last inequality follows by Markov's inequality, $\tilde{\mathbf{P}}\left(S_{l j} \geq 1\right) \leq \tilde{\mathbf{E}} S_{l j}$. Taking $\mathbf{E}^{\star}$-expected values in 32 we arrive to 31 since $\mathbf{P}^{\star}\left(S_{l j} \geq 1\right)=\mathbf{E}^{\star}\left(\tilde{\mathbf{P}}\left(S_{l j} \geq 1\right)\right)$. 
Now, 29 follows from $(31)$ and the fact that $\tilde{\mathbf{E}} S_{l j}=o_{P^{\star}}(1)$ for any realized values $Y_{1}, Y_{2}, X_{1}$. To show that the latter bound holds true we estimate

$$
\begin{aligned}
\tilde{\mathbf{E}} S_{00} & \leq \sum_{2 \leq i \leq m} \lambda_{i 1} \lambda_{i 2}=Y_{1} Y_{2} n^{-1} \hat{a}_{2}=o_{P^{\star}}(1), \\
\tilde{\mathbf{E}} S_{01} & \leq \sum_{3 \leq j \leq n} \sum_{2 \leq i_{1} \neq i_{2} \leq m} \lambda_{i_{1} 1} \lambda_{i_{1} j} \lambda_{i_{2} 2} \lambda_{i_{2} j} \leq Y_{1} Y_{2}\left(\hat{a}_{2}\right)^{2} \hat{b}_{2} n^{-1}=o_{P^{\star}}(1), \\
\tilde{\mathbf{E}} S_{k 0} & \leq \sum_{3 \leq j \leq n} \sum_{2 \leq i \leq m} \lambda_{i k} \lambda_{i j} \lambda_{1 j} \leq Y_{1} X_{1} \beta_{n}^{-1 / 2} \hat{a}_{2} \hat{b}_{2} n^{-1}=o_{P^{\star}}(1), \\
\tilde{\mathbf{E}} S_{k 0} & \leq \sum_{3 \leq j \leq n} \sum_{2 \leq i_{1}<i_{2} \leq m} \lambda_{i_{1} k} \lambda_{i_{1 j} j} \lambda_{i_{2} k} \lambda_{i_{2} j} \leq Y_{k}^{2}\left(\hat{a}_{2}\right)^{2} \hat{b}_{2} n^{-1}=o_{P^{\star}}(1) .
\end{aligned}
$$

Here we used the fact that $\mathbf{E} X_{1}^{2}<\infty$ implies $\hat{a}_{2}=O_{P}(1)$ and $\mathbf{E} Y_{1}<\infty$ implies $\hat{b}_{2} n^{-1}=o_{P}(1)$. Step 3. Here we prove (17). Denote

$$
\Delta_{1}=\mathbf{P}^{\star}\left(\mathcal{U}_{r, r_{1}, r_{2}}^{*}\right)-\mathbf{P}^{\star}\left(\mathcal{U}_{r, r_{1}, r_{2}}^{* *}\right), \quad \Delta_{2}=\mathbf{P}^{\star}\left(\mathcal{A}_{1}\right)-\lambda_{11} \lambda_{12} .
$$

From identities

$$
\mathbf{P}\left(\mathcal{U}_{r, r_{1}, r_{2}}^{*} \cap \mathcal{A}_{1}\right)=\mathbf{E}\left(\mathbf{P}^{\star}\left(\mathcal{U}_{r, r_{1}, r_{2}}^{*}\right) \mathbf{P}^{\star}\left(\mathcal{A}_{1}\right)\right), \quad \mathbf{P}\left(\mathcal{U}_{r, r_{1}, r_{2}}^{* *} \cap \mathcal{A}_{1}\right)=\mathbf{E}\left(\mathbf{P}^{\star}\left(\mathcal{U}_{r, r_{1}, r_{2}}^{* *}\right) \mathbf{P}^{\star}\left(\mathcal{A}_{1}\right)\right)
$$

we obtain

$$
\mathbf{P}\left(\mathcal{U}_{r, r_{1}, r_{2}}^{*} \cap \mathcal{A}_{1}\right)-\lambda_{11} \lambda_{12} \mathbf{P}\left(\mathcal{U}_{r, r_{1}, r_{2}}^{* *}\right)=\mathbf{E}\left(\Delta_{1} \mathbf{P}^{\star}\left(\mathcal{A}_{1}\right)\right)+\mathbf{E}\left(\Delta_{2} \mathbf{P}^{\star}\left(\mathcal{U}_{r, r_{1}, r_{2}}^{* *}\right)\right) .
$$

We shall show in Lemma 2 below that for any realized values $Y_{1}, Y_{2}, X_{1}$ we have $\Delta_{1}=o(1)$. This, together with the inequality $\mathbf{P}^{\star}\left(\mathcal{A}_{1}\right) \leq \lambda_{11} \lambda_{12}$ implies

$$
\mathbf{E}\left(\Delta_{1} \mathbf{P}^{\star}\left(\mathcal{A}_{1}\right)\right) \leq(n m)^{-1} \mathbf{E}\left(\Delta_{1} Y_{1} Y_{2} X_{1}^{2}\right)=o\left((n m)^{-1}\right),
$$

by Lebesgue's dominated convergence theorem. Furthermore, 24 implies

$$
\left|\mathbf{E}\left(\Delta_{2} \mathbf{P}^{\star}\left(\mathcal{U}_{r, r_{1}, r_{2}}^{* *}\right)\right)\right| \leq \mathbf{E}\left|\Delta_{2}\right| \leq(n m)^{-1} \mathbf{E} Y_{1} Y_{2} X_{1}^{2}\left(\overline{\mathbf{I}}_{11}+\overline{\mathbf{I}}_{12}\right)=o\left((n m)^{-1}\right) .
$$

Collecting (34) and (35) in (33) we obtain (17).

Proof of (ii). The proof (8) is similar to that of (7). It makes use the observation that the typical adjacency relation is witnessed by a single attribute. One difference is that now the size of the collection of attributes, prescribed to the typical vertex, tends to infinity as $m / n \rightarrow+\infty$ while the number of vertices sharing any given attribute tends to zero. As a consequence we obtain that $d_{12}=o_{P}(1)$.

The first several steps of the proof are the same as that of (7). Namely, relations (14), (15), (16) hold true as the argument of their proof remains valid for $m / n \rightarrow+\infty$. Further steps of the proof are a bit different. We show that

$$
\mathbf{P}\left(\mathcal{U}_{r, k_{1}-r, k_{2}-r}^{*} \cap \mathcal{A}_{1}\right) \simeq 0, \quad \text { for } \quad r=1,2, \ldots, k_{1} \wedge k_{2},
$$

and

$$
\mathbf{P}\left(\mathcal{U}_{0, k_{1}, k_{2}}^{*} \cap \mathcal{A}_{1}\right) \simeq \mathbf{P}\left(\left\{L_{1}=k_{1}, L_{2}=k_{2}\right\} \cap \mathcal{A}_{1}\right) .
$$

Finally, we show that

$$
\mathbf{P}\left(\left\{L_{1}=k_{1}, L_{2}=k_{2}\right\} \cap \mathcal{A}_{1}\right) \simeq \mathbf{E}\left(\lambda_{11} \lambda_{12} \mathbf{P}^{\star}\left(\mathcal{U}_{k_{1}, k_{2}}^{* * *}\right)\right) .
$$


Now the simple identity

$$
a_{2}^{-1} b_{1}^{-2} n m \mathbf{E}\left(\lambda_{11} \lambda_{12} \mathbf{P}^{\star}\left(\mathcal{U}_{k_{1}, k_{2}}^{* * *}\right)\right)=\tilde{p}\left(k_{1}+1\right) \tilde{p}\left(k_{2}+1\right)
$$

completes the proof of (8). It remains to prove (36), (37), (38).

Let us prove (36), (37). Denote $R^{\prime}=\mathbf{P}\left(\left\{L_{0} \geq 1\right\} \cap \mathcal{A}_{1}\right)$. Relations (36), (37) follow from inequalities

$$
\begin{aligned}
& \mathbf{P}\left(\mathcal{U}_{r, k_{1}-r, k_{2}-r}^{*} \cap \mathcal{A}_{1}\right) \leq R^{\prime}, \quad r=1,2, \ldots, \\
& 0 \leq \mathbf{P}\left(\left\{L_{1}=k_{1}, L_{2}=k_{2}\right\} \cap \mathcal{A}_{1}\right)-\mathbf{P}\left(\mathcal{U}_{0, k_{1}, k_{2}}^{*} \cap \mathcal{A}_{1}\right) \leq R^{\prime},
\end{aligned}
$$

and the bound $R^{\prime}=o\left((n m)^{-1}\right)$. To prove the latter bound we write

$$
R^{\prime}=\mathbf{E}\left(\mathbf{P}^{\star}\left(L_{0} \geq 1\right) \mathbf{P}^{\star}\left(A_{1}\right)\right) \leq(n m)^{-1} \mathbf{E}\left(Y_{1} Y_{2} X_{1}^{2} \mathbf{P}^{\star}\left(L_{0} \geq 1\right)\right)=o\left((n m)^{-1}\right) .
$$

Here we used inequalities $\mathbf{P}^{\star}\left(\mathcal{A}_{1}\right) \leq \lambda_{11} \lambda_{12} \leq(n m)^{-1} Y_{1} Y_{2} X_{1}^{2}$ and the bound $\mathbf{E}\left(Y_{1} Y_{2} X_{1}^{2} \mathbf{P}^{\star}\left(L_{0} \geq 1\right)\right)=o(1)$, which follows by Lebesgue's dominated convergence theorem, since

$$
\mathbf{P}^{\star}\left(L_{0} \geq 1\right) \leq \mathbf{E}^{\star} L_{0} \leq \sum_{3 \leq j \leq n} \lambda_{1 j}=b_{1} X_{1}((n-2) / n) \beta_{n}^{-1 / 2}=o(1) .
$$

Let us prove (38). The proof is similar to that of (17). We write

$$
\mathbf{P}\left(\left\{L_{1}=k_{1}, L_{2}=k_{2}\right\} \cap \mathcal{A}_{1}\right)-\lambda_{11} \lambda_{12} \mathbf{P}\left(\mathcal{U}_{k_{1}, k_{2}}^{* * *}\right)=\mathbf{E}\left(\Delta_{3} \mathbf{P}^{\star}\left(\mathcal{A}_{1}\right)\right)+\mathbf{E}\left(\Delta_{2} \mathbf{P}^{\star}\left(\mathcal{U}_{k_{1}, k_{2}}^{* * *}\right)\right),
$$

where

$$
\Delta_{2}=\mathbf{P}^{\star}\left(\mathcal{A}_{1}\right)-\lambda_{11} \lambda_{12}, \quad \Delta_{3}=\mathbf{P}^{\star}\left(L_{1}=k_{1}, L_{2}=k_{2}\right)-\mathbf{P}^{\star}\left(\mathcal{U}_{k_{1}, k_{2}}^{* * *}\right)
$$

and show that both summands in the right of $(42)$ are $o\left((n m)^{-1}\right)$, cf. proof of (17) above. In fact, the only thing that remains to show is that for any realized values $Y_{1}, Y_{2}, X_{1}$ we have $\Delta_{3}=o(1)$. This is shown in Lemma 3 below.

Proof of Remark 1. Let us prove (i). Lemma 4 implies

$$
\mathbf{P}\left(\Lambda_{0}=r\right) \approx c_{1}^{*} r^{-\varkappa}, \quad \text { where } \quad c_{1}^{*}=c\left(b_{1} \beta^{-1 / 2}\right)^{\varkappa-1} .
$$

Consequently,

$$
\mathbf{P}\left(\tau_{1}=r\right) \approx c a_{1}^{-1}\left(b_{1} \beta^{-1 / 2}\right)^{\varkappa-2} r^{1-\varkappa} .
$$

From the latter relation we conclude that the sequence of probabilities $\left\{\mathbf{P}\left(\tau_{1}=r\right)\right\}_{r \geq 0}$ is longtailed and sub-exponential, that is, it satisfies conditions of Theorem 4.30 of [9]. This theorem implies $\mathbf{P}\left(\sum_{1<i<\Lambda_{1}} \tau_{i}=r\right) \approx \mathbf{P}\left(\tau_{1}=r\right) \mathbf{E} \Lambda_{1}$, thus completing the proof.

Let us prove (ii). We observe that $\tau_{1}$ has Poisson distribution with mean $\lambda_{0}=x b_{1} \beta^{-1 / 2}$. Hence, given $\Lambda_{1}$, the random variable $d_{*}$ has Poisson distribution with mean $\lambda_{0} \Lambda_{1}$. Now statement (iii) of Lemma 4 implies that $\mathbf{P}\left(\Lambda_{1}=r\right) \approx c_{*} r^{-\varkappa}$, where $c_{*}=c\left(a_{1} \beta^{1 / 2}\right)^{\varkappa-1}$. Next, we apply statement (iii) of Lemma 4 once again and obtain $\mathbf{P}\left(d_{*}=r\right) \approx c_{*} \lambda_{0}^{\varkappa-1} r^{-\varkappa}$.

Proof of Remark 2. The proof is standard. We present it here for reader's convenience. Let $\left(v_{1}^{\prime}, v_{2}^{\prime}\right)$ denote an ordered pair of distinct vertices drawn uniformly at random and let $\mathbf{P}^{\prime}$ denote the conditional probability given all the random variables considered, but $\left(v_{1}^{\prime}, v_{2}^{\prime}\right)$. We have

$$
\mathbf{P}\left(d\left(v_{1}\right)=r \mid v_{1} \sim v_{2}\right)=\frac{\mathbf{P}\left(d\left(v_{1}\right)=r, v_{1} \sim v_{2}\right)}{\mathbf{P}\left(v_{1} \sim v_{2}\right)}=\frac{\mathbf{P}\left(d\left(v_{1}^{\prime}\right)=r, v_{1}^{\prime} \sim v_{2}^{\prime}\right)}{\mathbf{P}\left(v_{1} \sim v_{2}\right)} .
$$


The denominator is evaluated in (18): We have

$$
\mathbf{P}\left(\left\{v_{1} \sim v_{2}\right\}\right)=n^{-1} a_{2} b_{1}^{2}+o\left(n^{-1}\right)=n^{-1} \mathbf{E} \Lambda_{3}+o\left(n^{-1}\right) .
$$

In the last step we used the simple identities $\mathbf{E} \Lambda_{3}=\mathbf{E} \lambda_{3}=a_{2} b_{1}^{2}$. In order to evaluate the numerator we combine identities

$$
\begin{aligned}
& \mathbf{P}^{\prime}\left(d\left(v_{1}^{\prime}\right)=r, v_{1}^{\prime} \sim v_{2}^{\prime}\right)=\mathbf{P}^{\prime}\left(v_{1}^{\prime} \sim v_{2}^{\prime} \mid d\left(v_{1}^{\prime}\right)=r\right) \mathbf{P}^{\prime}\left(d\left(v_{1}^{\prime}\right)=r\right)=\frac{r}{n-1} \mathbf{P}^{\prime}\left(d\left(v_{1}^{\prime}\right)=r\right) \\
& \mathbf{P}\left(d\left(v_{1}^{\prime}\right)=r, v_{1}^{\prime} \sim v_{2}^{\prime}\right)=\mathbf{E}\left(\mathbf{P}^{\prime}\left(d\left(v_{1}^{\prime}\right)=r, v_{1}^{\prime} \sim v_{2}^{\prime}\right)\right) .
\end{aligned}
$$

We obtain $\mathbf{P}\left(d\left(v_{1}^{\prime}\right)=r, v_{1}^{\prime} \sim v_{2}^{\prime}\right)=(r /(n-1)) \mathbf{P}\left(d\left(v_{1}\right)=r\right)$. Hence, by 45),

$$
\mathbf{P}\left(d\left(v_{1}\right)=r \mid v_{1} \sim v_{2}\right)=r \mathbf{P}\left(d\left(v_{1}\right)=r\right)\left(\mathbf{E} \Lambda_{3}\right)^{-1}+o(1) .
$$

Now, the statement (ii) of Theorem 1 completes the proof of $(9)$.

Proof of Theorem 3. (i) follows from the relation $\mathbf{P}\left(\Lambda_{3}=r\right) \approx c\left(a_{2} b_{1}\right)^{\varkappa-1} r^{-\varkappa}$, see Lemma 4 . In the proof of (ii) and (iii) we assume that $k_{1} \leq k_{2}$. We recall that $q_{i}$ is defined in (6) and introduce the notation

$$
S_{A}=\sum_{r \in A}(r+1)(r+2) \mathbf{P}\left(\Lambda_{0}=r+2\right) q_{k_{1}-r} q_{k_{2}-r}, \quad A \subset\left[0, k_{1}\right] .
$$

Let us prove (ii). We observe that $\mathbf{E}\left(e^{a Y_{1}}\right)<\infty$ implies that $\mathbf{E} Y_{1} e^{a^{\prime} \Lambda_{1}}<\infty$ for some $a^{\prime}>0$. Using this observation and the fact that the sequence of probabilities $\left\{\mathbf{P}\left(\tau_{1}=r\right)\right\}_{r \geq 0}$ is longtailed and sub-exponential (see (44) and 9]) we show that

$$
\mathbf{E}\left(Y_{1} \mathbf{P}\left(d_{Y_{1}}^{*}=r \mid Y_{1}\right)\right) \approx\left(\mathbf{E}\left(Y_{1} \Lambda_{1}\right)\right) \mathbf{P}\left(\tau_{1}=r\right) .
$$

The proof of (46) is much the same as that of Theorem 4.30 in [9]. Next, we invoke in (46) the identity $\mathbf{E}\left(Y_{1} \Lambda_{1}\right)=\mathbf{E}\left(Y_{1} \lambda_{1}\right)=a_{1} b_{2} \beta^{1 / 2}$ and 44 , and obtain

$$
\mathbf{E}\left(Y_{1} \mathbf{P}\left(d_{Y_{1}}^{*}=r \mid Y_{1}\right)\right) \approx c_{2}^{*} r^{1-\varkappa}, \quad \text { where } \quad c_{2}^{*}=c b_{1}^{\varkappa-2} b_{2} \beta^{(3-\varkappa) / 2} .
$$

Hence we have $q_{r} \approx c_{2}^{*} r^{1-\varkappa}$ and $\mathbf{P}\left(\Lambda_{0}=r\right) \approx c_{1}^{*} r^{-\varkappa}$, see $(43)$.

Now we are ready to prove (11). Let $\varepsilon=\ln \left(k_{1} \wedge\left(k_{2}-k_{1}\right)\right)$ for $k_{2}-k_{1} \rightarrow+\infty$, and $\varepsilon=\ln k_{1}$ otherwise. Split $S_{\left[0, k_{1}\right]}=S_{A_{1}}+S_{A_{2}}+S_{A_{3}}$, where

$$
A_{1}=\left[0, k_{1} / 2\right], \quad A_{2}=\left(k_{1} / 2, k_{1}-\varepsilon\right], \quad A_{3}=\left(k_{1}-\varepsilon, k_{1}\right] .
$$

In the remaining part of the proof we shall show that $S_{A_{1}}, S_{A_{2}}$ are negligibly small compared to $S_{A_{3}}$ and determine the first order asymptotics of $S_{A_{3}}$ as $k_{1}, k_{2} \rightarrow+\infty$. We have for some $\bar{c}>0$ (independent of $k_{1}, k_{2}$ )

$$
S_{A_{1}} \leq \bar{c} \sum_{i \in A_{1}} \frac{1}{\left(k_{1}-i\right)^{\varkappa-1}} \frac{1}{\left(k_{2}-i\right)^{\varkappa-1}} \frac{1}{(1+i)^{\varkappa-2}}=O\left(k_{1}^{4-2 \varkappa} k_{2}^{1-\varkappa}(1+\Delta)\right) .
$$

Here $\Delta=\ln n$ for $\varkappa=3$ and $\Delta=0$ otherwise. Furthermore, for $k_{2}-k_{1}$ bounded we have

$$
S_{A_{2}} \leq \frac{\bar{c}}{k_{1}^{\varkappa-2}} \sum_{i \in A_{2}} \frac{1}{\left(k_{1}-i\right)^{2 \varkappa-2}}=O\left(\varepsilon^{3-2 \varkappa} k_{1}^{2-\varkappa}\right)=o\left(k_{1}^{2-\varkappa}\right) .
$$


For $k_{2}-k_{1} \rightarrow+\infty$ we have

$$
S_{A_{2}} \leq \frac{\bar{c}}{k_{1}^{\varkappa-2}} \sum_{i \in A_{2}} \frac{1}{\left(k_{1}-i\right)^{\varkappa-1}} \frac{1}{\left(k_{2}-i\right)^{\varkappa-1}} \leq \frac{\bar{c}}{k_{1}^{\varkappa-2}\left(k_{2}-k_{1}\right)^{\varkappa-1}} \sum_{i \in A_{2}} \frac{1}{\left(k_{1}-i\right)^{\varkappa-1}} .
$$

Since $\sum_{i \in A_{2}} \frac{1}{\left(k_{1}-i\right)^{\varkappa-1}}=O\left(\varepsilon^{2-\varkappa}\right)=o(1)$ we obtain $S_{A_{2}}=o\left(k_{1}^{2-\varkappa}\left(k_{2}-k_{1}\right)^{1-\varkappa}\right)$.

Finally, using the approximation $(i+1)(i+2) \mathbf{P}\left(\Lambda_{0}=i+2\right) \approx c_{1}^{*} k_{1}^{2-\varkappa}$ uniformly in $i \in A_{3}$ we obtain for $k_{2}-k_{1} \rightarrow+\infty$

$$
S_{A_{3}}=\frac{c_{1}^{*}(1+o(1))}{k_{1}^{\varkappa-2}} \sum_{i \in A_{3}} q_{k_{1}-i} q_{k_{2}-i} \approx \frac{c_{1}^{*}}{k_{1}^{\varkappa-2}} \frac{c_{2}^{*}}{\left(k_{2}-k_{1}\right)^{\varkappa-1}} \sum_{i \in A_{3}} q_{k_{1}-i} \approx \frac{c_{1}^{*} c_{2}^{*} b_{1}}{k_{1}^{\varkappa-2}\left(k_{2}-k_{1}\right)^{\varkappa-1}} .
$$

Here we used $\sum_{r \geq 0} q_{r}=b_{1}$. Similarly, in the case where $k_{2}-k_{1}=k$ for some fixed $k$ we have

$$
S_{A_{3}}=\frac{c_{1}^{*}(1+o(1))}{k_{1}^{\varkappa-2}} \sum_{i \in A_{3}} q_{k_{1}-i} q_{k_{2}-i} \approx \frac{c_{1}^{*} c_{3, k}^{*}}{k_{1}^{\varkappa-2}}, \quad \text { where } \quad c_{3, k}^{*}=\sum_{i \geq 0} q_{i} q_{k+i} .
$$

Let us prove (iii). We observe that $\Lambda_{0}$ has the Poisson distribution with (non-random) mean $\lambda_{0}$. Using the identity $(r+1)(r+2) \mathbf{P}\left(\Lambda_{0}=r+2\right)=\lambda_{0}^{2} \mathbf{P}\left(\Lambda_{0}=r\right)$ we write

$$
\begin{aligned}
& S_{\left[0, k_{1}\right]}=\lambda_{0}^{2} \sum_{r=0}^{k_{1}} \mathbf{P}\left(\Lambda_{0}=r\right) q_{k_{1}-r} q_{k_{2}-r}=\lambda_{0}^{2} \mathbf{E}\left(q_{k_{1}-\Lambda_{0}} q_{k_{2}-\Lambda_{0}}\right)=\lambda_{0}^{2}\left(J_{1}+J_{2}\right), \\
& J_{1}=\mathbf{E}\left(q_{k_{1}-\Lambda_{0}} q_{k_{2}-\Lambda_{0}}\right) \mathbb{I}_{\left\{\Lambda_{0}<\sqrt{k_{1}}\right\}}, \quad J_{2}=\mathbf{E}\left(q_{k_{1}-\Lambda_{0}} q_{k_{2}-\Lambda_{0}}\right) \mathbb{I}_{\left\{\sqrt{k_{1}} \leq \Lambda_{0} \leq k_{1}\right\}} .
\end{aligned}
$$

Next, combining the fast decay of Poisson tail probability $\mathbf{P}\left(\Lambda_{0}>t\right)$ as $t \rightarrow+\infty$ with the relation, which is shown below,

$$
q_{r} \approx c_{0} r^{1-\varkappa}, \quad c_{0}=c\left(x^{2} b_{1}\right)^{\varkappa-2},
$$

we estimate $J_{1}=(1+o(1)) c_{0}^{2}\left(k_{1} k_{2}\right)^{1-\varkappa}$ and $J_{2}=o\left(\left(k_{1} k_{2}\right)^{1-\varkappa}\right.$. We obtain that $S_{[0,1]}=(1+$ $o(1)) \lambda_{0}^{2} J_{1}$. Now the identity $p_{\beta}\left(k_{1}, k_{2}\right)=\beta b_{1}^{-4} x^{-2} S_{\left[0, k_{1}\right]}$ completes the proof of 12 .

Let us prove 47). Since $\tau_{1}$ has Poisson distribution with mean $\lambda_{0}=x b_{1} \beta^{-1 / 2}$, we obtain

$$
\begin{aligned}
\mathbf{P}\left(d_{Y_{1}}^{*}=k \mid Y_{1}=y\right) & =\mathbf{E}\left(\mathbf{P}\left(d_{Y_{1}}^{*}=k \mid \Lambda_{1}, Y_{1}=y\right) \mid Y_{1}=y\right) \\
& =\mathbf{E}\left(e^{-\lambda_{0} \Lambda_{1}} \frac{\left(\lambda_{0} \Lambda_{1}\right)^{k}}{k !} \mid Y_{1}=y\right) \\
& =\sum_{i \geq 0} e^{-\lambda_{0} i} \frac{\left(\lambda_{0} i\right)^{k}}{k !} e^{-B y} \frac{(B y)^{i}}{i !} .
\end{aligned}
$$

Here we denote $B=x \beta^{1 / 2}$. After we write the product $y \mathbf{P}\left(d_{y}^{*}=k\right)$ in the form

$$
\sum_{i \geq 0} e^{-\lambda_{0} i} \frac{\left(\lambda_{0} i\right)^{k}}{k !} e^{-B y} \frac{(B y)^{i+1}}{(i+1) !} \frac{i+1}{B}=\mathbf{E}\left(e^{-\lambda_{0}\left(\Lambda_{1}-1\right)} \frac{\left(\lambda_{0}\left(\Lambda_{1}-1\right)\right)^{k}}{k !} \frac{\Lambda_{1}}{B} \mathbb{I}_{\left\{\Lambda_{1} \geq 1\right\}} \mid Y_{1}=y\right)
$$

we obtain the following expression for the expectation $q_{k}=\mathbf{E}\left(Y_{1} \mathbf{P}\left(d_{Y_{1}}^{*}=k \mid Y_{1}\right)\right)$

$$
q_{k}=\frac{\mathbf{P}\left(\Lambda_{1} \geq 1\right)}{B}\left(I_{1, k}+I_{2, k}\right), \quad I_{1, k}=\mathbf{E}\left(e^{-\lambda_{0} Z} \frac{\left(\lambda_{0} Z\right)^{k}}{k !}\right), \quad I_{2, k}=\mathbf{E}\left(Z e^{-\lambda_{0} Z} \frac{\left(\lambda_{0} Z\right)^{k}}{k !}\right) .
$$


Here $Z$ denotes a random variable with the distribution

$$
\mathbf{P}(Z=r)=\mathbf{P}\left(\Lambda_{1}=r+1\right) / \mathbf{P}\left(\Lambda_{1} \geq 1\right), \quad r=0,1, \ldots
$$

We note that

$$
\mathbf{P}(Z=r) \approx c^{\prime} r^{-\varkappa}, \quad \text { where } \quad c^{\prime}=c B^{\varkappa-1} / \mathbf{P}\left(\Lambda_{1} \geq 1\right) .
$$

Indeed, 49 follows from the relation $\mathbf{P}\left(\Lambda_{1}=r\right) \approx c B^{\varkappa-1} r^{-\varkappa}$, which is a simply consequence of the property $\mathcal{P}_{c, \varkappa}$ of the distribution of $Y_{1}$, see Lemma 4. Next, we show that

$$
I_{1, k} \approx c^{\prime} \lambda_{0}^{\varkappa-1} k^{-\varkappa}, \quad I_{2, k} \approx c^{\prime} \lambda_{0}^{\varkappa-2} k^{1-\varkappa} .
$$

The first relation follows from $(49)$, by Lemma 4. The second relation follows from the first one via the simple identity $I_{2, k}=(k+1) \lambda_{0}^{-1} I_{1, k+1}$. Finally invoking $(50)$ in 480 we obtain $(47)$.

\section{Appendix A}

Here we prove the bounds $\Delta_{1}=o(1)$ and $\Delta_{3}=o(1)$, see $(33)$ and 442$)$ above. In the proof we apply and further extend the approach of [4]. An important tool used below is the following inequality referred to as Le Cam's lemma, see e.g., [20].

Lemma 1. Let $S=\mathbb{I}_{1}+\mathbb{I}_{2}+\cdots+\mathbb{I}_{n}$ be the sum of independent random indicators with probabilities $\mathbf{P}\left(\mathbb{I}_{i}=1\right)=p_{i}$. Let $\Lambda$ be Poisson random variable with mean $p_{1}+\cdots+p_{n}$. The total variation distance between the distributions $P_{S}$ of $P_{\Lambda}$ of $S$ and $\Lambda$

$$
\sup _{A \subset\{0,1,2 \ldots\}}|\mathbf{P}(S \in A)-\mathbf{P}(\Lambda \in A)|=\frac{1}{2} \sum_{k \geq 0}|\mathbf{P}(S=k)-\mathbf{P}(\Lambda=k)| \leq \sum_{i} p_{i}^{2} .
$$

Lemma 2. Assume that conditions of part (i) of Theorem 2 are satisfied. Then for any realized values $Y_{1}, Y_{2}, X_{1}$ and any integers $r, r_{1}, r_{2} \geq 0$ we have

$$
\mathbf{P}^{\star}\left(\mathcal{U}_{r, r_{1}, r_{2}}^{*}\right)-\mathbf{P}^{\star}\left(\mathcal{U}_{r, r_{1}, r_{2}}^{* *}\right)=o(1) .
$$

Proof of Lemma 2, Denote

$$
\mathbf{L}^{(0)}=\left(L_{0}^{(0)}, L_{1}^{(0)}, L_{2}^{(0)}\right):=\left(L_{0}, L_{1}, L_{2}\right), \quad \mathbf{L}^{(4)}=\left(L_{0}^{(4)}, L_{1}^{(4)}, L_{2}^{(4)}\right):=\left(\Lambda_{0}, d_{Y_{1}}^{*}, d_{Y_{2}}^{*}\right) .
$$

In the proof we construct random vectors $\mathbf{L}^{(h)}=\left(L_{0}^{(h)}, L_{1}^{(h)}, L_{2}^{(h)}\right), h=1,2,3$, defined on the same probability space as $Y_{1}, Y_{2}, X_{1}$ such that for any realized values $Y_{1}, Y_{2}, X_{1}$ we have

$$
\begin{aligned}
& d_{T V}^{\star}\left(\mathbf{L}^{(h)}, \mathbf{L}^{(h+1)}\right)=o(1), \quad h=0,1, \\
& \mathbf{P}^{\star}\left(\mathbf{L}^{(h)}=\left(r, r_{1}, r_{2}\right)\right)-\mathbf{P}^{\star}\left(\mathbf{L}^{(h+1)}=\left(r, r_{1}, r_{2}\right)\right)=o(1), \quad h=2,3,
\end{aligned}
$$

for any integers $r, r_{1}, r_{2} \geq 0$. We note that (53), (54) imply (52).

Let us define random vectors $\mathbf{L}^{(h)}, h=1,2,3$. Given $X, Y$, let $\left\{\eta_{k i}\right\}_{i=2}^{m}, k=1,2$ and $\left\{\xi_{h i}\right\}_{i=1}^{m}$, $h=2,3$, be sequences of Poisson random variables which are (conditionally, given $X, Y$ ) independent within each sequence and have mean values

$$
\tilde{\mathbf{E}} \eta_{k i}=\lambda_{k i}, \quad \tilde{\mathbf{E}} \xi_{2 i}=\sum_{3 \leq j \leq n} \lambda_{i j}, \tilde{\mathbf{E}} \xi_{3 i}=\frac{b_{1}}{\sqrt{\beta}} X_{i}
$$


In addition, we assume that (conditionally, given $X, Y$ ) the sequences $\left\{\eta_{k i}\right\}_{i=2}^{m}, k=1,2$, are independent and they are independent of the sequences $\left\{\xi_{h i}\right\}_{i=1}^{m}, h=2,3,4$. Denote for $k=1,2$ and $h=2,3$

$$
L_{0}^{(1)}=u_{1}, \quad L_{0}^{(h)}=\xi_{h 1}, \quad L_{k}^{(1)}=\sum_{2 \leq i \leq m} \eta_{k i} u_{i}, \quad L_{k}^{(h)}=\sum_{2 \leq i \leq m} \eta_{k i} \xi_{h i} .
$$

Let us prove $(53)$ for $h=0$. In the proof we use the following simple inequalities. Let $\tilde{d}_{T V}^{*}(\zeta, \theta)$ denote the total variation distance between the conditional distributions of random variables/vectors $\zeta$ and $\theta$ given $X, Y, u_{1}, \ldots, u_{m}$. Then we have

$$
\tilde{d}_{T V}(\zeta, \theta) \leq \tilde{\mathbf{E}} \tilde{d}_{T V}^{*}(\zeta, \theta), \quad d_{T V}^{\star}(\zeta, \theta) \leq \mathbf{E}^{\star} \tilde{d}_{T V}(\zeta, \theta) \leq \mathbf{E}^{\star} \tilde{d}_{T V}^{*}(\zeta, \theta) .
$$

Introduce random variables for $k=1,2$ and $t=1, \ldots, m$

$$
L_{k}^{[t]}=\sum_{2 \leq i \leq t} \mathbb{I}_{i k} u_{i}+\sum_{t+1 \leq i \leq m} \eta_{k i} u_{i}
$$

Note that $L_{k}^{[1]}=L_{k}^{(1)}$ and $L_{k}^{[m]}=L_{k}^{(0)}$. We have, by the triangle inequality,

$$
\tilde{d}_{T V}^{*}\left(L_{k}^{[1]}, L_{k}^{[m]}\right) \leq \sum_{2 \leq t \leq m} \tilde{d}_{T V}^{*}\left(L_{k}^{[t-1]}, L_{k}^{[t]}\right) \leq \sum_{t=2}^{m} \lambda_{t k}^{2} .
$$

Here we estimated each summand

$$
\tilde{d}_{T V}^{*}\left(L_{k}^{[t-1]}, L_{k}^{[t]}\right) \leq \tilde{d}_{T V}^{*}\left(\mathbb{I}_{t k}, \eta_{k t}\right) \leq \lambda_{t k}^{2} .
$$

In the first inequality of 56 we use the fact that $L_{k}^{[t-1]}$ and $L_{k}^{[t]}$ only differ in the $t$-th summand. The second inequality is trivial for $\lambda_{t k} \geq 1$ and it follows by Le Cam's inequality, see Lemma 1 , for $\lambda_{t k}<1$.

Next, we use the fact that given $X, Y, u_{1}, \ldots, u_{m}$ the random vectors $\mathbf{L}^{(0)}$ and $\mathbf{L}^{(1)}$ have conditionally independent marginals. In particular, we can apply the triangle inequality

$$
\tilde{d}_{T V}^{*}\left(\mathbf{L}^{(0)}, \mathbf{L}^{(1)}\right) \leq \sum_{0 \leq k \leq 2} \tilde{d}_{T V}^{*}\left(L_{k}^{(0)}, L_{k}^{(1)}\right) \leq \sum_{2 \leq t \leq m}\left(\lambda_{t 1}^{2}+\lambda_{t 2}^{2}\right)
$$

In the last step we use $\tilde{d}_{T V}^{*}\left(L_{0}^{(0)}, L_{0}^{(1)}\right)=0$. Finally, 55 implies

$$
d_{T V}^{\star}\left(\mathbf{L}^{(0)}, \mathbf{L}^{(1)}\right) \leq \mathbf{E}^{\star} \sum_{2 \leq t \leq m}\left(\lambda_{t 1}^{2}+\lambda_{t 2}^{2}\right)=\frac{2}{n} \frac{m-2}{m} a_{2}\left(Y_{1}^{2}+Y_{2}^{2}\right)=o(1) .
$$

Let us prove $(53)$ for $h=1$. We note that conditionally, given $X, Y$, the random variable $L_{0}^{(1)}$ is independent of $\left(L_{1}^{(1)}, L_{2}^{(1)}\right)$, and $L_{0}^{(2)}$ is independent of $\left(L_{1}^{(2)}, L_{2}^{(2)}\right)$. Hence, we have, by triangle inequality,

$$
\tilde{d}_{T V}\left(\mathbf{L}^{(1)}, \mathbf{L}^{(2)}\right) \leq \tilde{d}_{T V}\left(L_{0}^{(1)}, L_{0}^{(2)}\right)+\tilde{d}_{T V}\left(\left(L_{1}^{(1)}, L_{2}^{(1)}\right),\left(L_{1}^{(2)}, L_{2}^{(2)}\right)\right)=: Z_{1}+Z_{2} .
$$

We shall show that $\mathbf{E}^{\star} Z_{i}=o(1), i=1,2$. These bounds combined with the second inequality of 55 imply $d_{T V}^{\star}\left(\mathbf{L}^{(1)}, \mathbf{L}^{(2)}\right)=o(1)$. 
We firstly estimate $Z_{1}$. We have

$$
Z_{1}=\tilde{d}_{T V}\left(u_{1}, \xi_{21}\right) \leq \sum_{3 \leq j \leq n} \lambda_{1 j}^{2}=\beta_{n}^{-1} X_{1}^{2} \hat{b}_{2} n^{-1} .
$$

Indeed, for $\max _{j} \lambda_{1 j}<1$ this inequality follows by Lemma 1. Otherwise the inequality is trivial, since the total variation distance is always less than or equal to 1 . Next, we use the fact that $\mathbf{E} Y_{1}<\infty$ implies $\hat{b}_{2} n^{-1}=o_{P}(1)$. We obtain $Z_{1}=o_{P^{\star}}(1)$. This bound together with the inequality $Z_{1} \leq 1$ implies $\mathbf{E}^{\star} Z_{1}=o(1)$.

We secondly estimate $Z_{2}$. Introduce random variables for $k=1,2$ and $t=1, \ldots, m$

$$
L_{k}^{\{t\}}=\sum_{2 \leq i \leq t} \eta_{k i} u_{i}+\sum_{t+1 \leq i \leq m} \eta_{k i} \xi_{2 i} .
$$

Note that $L_{k}^{\{1\}}=L_{k}^{(2)}$ and $L_{k}^{\{m\}}=L_{k}^{(1)}$. We apply triangle inequality

$$
\tilde{d}_{T V}\left(\left(L_{1}^{\{m\}}, L_{2}^{\{m\}}\right),\left(L_{1}^{\{1\}}, L_{2}^{\{1\}}\right)\right) \leq \sum_{t=2}^{m} \tilde{d}_{T V}\left(\left(L_{1}^{\{t-1\}}, L_{2}^{\{t-1\}}\right),\left(L_{1}^{\{t\}}, L_{2}^{\{t\}}\right)\right)
$$

and estimate each summand as follows

$$
\begin{aligned}
\tilde{d}_{T V}\left(\left(L_{1}^{\{t-1\}}, L_{2}^{\{t-1\}}\right),\left(L_{1}^{\{t\}}, L_{2}^{\{t\}}\right)\right) & \leq \tilde{d}_{T V}\left(\left(u_{t} \eta_{t 1}, u_{t} \eta_{t 2}\right),\left(\xi_{2 t} \eta_{t 1}, \xi_{2 t} \eta_{t 2}\right)\right) \\
& \leq \tilde{\mathbf{P}}\left(\left(\eta_{t 1}, \eta_{t 2}\right) \neq(0,0)\right) \tilde{d}_{T V}\left(u_{t}, \xi_{2 t}\right) \\
& \leq\left(\lambda_{t 1}+\lambda_{t 2}\right) \min \left\{1, \beta_{n}^{-1} X_{t}^{2} \hat{b}_{2} n^{-1}\right\} .
\end{aligned}
$$

In 59 we use the fact that $L_{k}^{\{t-1\}}$ and $L_{k}^{\{t\}}$ only differ in the $t$-th summand. In 60 we first estimate the total variation distance between conditional distributions of $\left(u_{t} \eta_{t 1}, u_{t} \eta_{t 2}\right)$ and $\left(\xi_{2 t} \eta_{t 1}, \xi_{2 t} \eta_{t 2}\right)$ given $\eta_{t 1}, \eta_{t 2}, X, Y$ from above by $\mathbb{I}_{\left\{\left(\eta_{t 1}, \eta_{t 2}\right) \neq(0,0)\right\}} \tilde{d}_{T V}\left(u_{t}, \xi_{2 t}\right)$ and then take the expected value $\tilde{\mathbf{E}}$. In 61 we estimate $\tilde{\mathbf{P}}\left(\left(\eta_{t 1}, \eta_{t 2}\right) \neq(0,0)\right) \leq \lambda_{t 1}+\lambda_{t 2}$. This bound is trivial for $\lambda_{t 1}+\lambda_{t 2} \geq 1$. Otherwise it follows from the inequalities

$$
\tilde{\mathbf{P}}\left(\left(\eta_{t 1}, \eta_{t 2}\right) \neq(0,0)\right) \leq \tilde{\mathbf{P}}\left(\eta_{t 1} \neq 0\right)+\tilde{\mathbf{P}}\left(\eta_{t 2} \neq 0\right)=e^{0}-e^{-\lambda_{t 1}}+e^{0}-e^{-\lambda_{t 2}} \leq \lambda_{t 1}+\lambda_{t 2} .
$$

$\operatorname{In} 61$ we invoke the inequality $\tilde{d}_{T V}\left(u_{t}, \xi_{2 t}\right) \leq \min \left\{1, \beta_{n}^{-1} X_{t}^{2} \hat{b}_{2} n^{-1}\right\}$, cf. (57) above.

Next, using the identities $L_{k}^{\{1\}}=L_{k}^{(2)}$ and $L_{k}^{\{m\}}=L_{k}^{(1)}$ we obtain from (58), 61 that

$$
Z_{2} \leq \sum_{t=2}^{m}\left(\lambda_{t 1}+\lambda_{t 2}\right) \min \left\{1, \frac{X_{t}^{2} \hat{b}_{2}}{\beta_{n} n}\right\}=\left(Y_{1}+Y_{2}\right) \beta_{n}^{1 / 2} \sum_{t=2}^{m} \frac{X_{t}}{m}\left\{1, \frac{X_{t}^{2} \hat{b}_{2}}{\beta_{n} n}\right\} .
$$

Finally, we have,

$$
\mathbf{E}^{\star} Z_{2}=\beta^{1 / 2}\left(Y_{1}+Y_{2}\right) \mathbf{E}^{\star} X_{2}\left\{1, X_{2}^{2} \hat{b}_{2} \beta_{n}^{-1} n^{-1}\right\}=\beta^{1 / 2}\left(Y_{1}+Y_{2}\right) \mathbf{E} X_{2}\left\{1, X_{2}^{2} \hat{b}_{2} \beta_{n}^{-1} n^{-1}\right\}=o(1),
$$

since $\hat{b}_{2}$ is independent of $X_{2}$ and $\mathbf{E} X_{2}<\infty$, and $\hat{b}_{2} n^{-1}=o_{P}(1)$.

Let us prove (54) for $h=2$. Given $X, Y$, let $\left\{\xi_{i}\right\}_{i=1}^{m},\left\{\Delta_{i}^{\prime}\right\}_{i=1}^{m},\left\{\Delta_{i}^{\prime \prime}\right\}_{i=1}^{m}$ be independent Poisson random variables, which are independent of $\left\{\eta_{i 1}\right\}_{i=2}^{m},\left\{\eta_{i 2}\right\}_{i=2}^{m}$, and have mean values

$$
\tilde{\mathbf{E}} \xi_{i}=b X_{i}, \quad \tilde{\mathbf{E}} \Delta_{i}^{\prime}=\delta^{\prime} X_{i}, \quad \tilde{\mathbf{E}} \Delta_{i}^{\prime \prime}=\delta^{\prime \prime} X_{i}, \quad 1 \leq i \leq m .
$$

Here $\delta^{\prime}=\hat{b}_{1} \beta_{n}^{-1 / 2}-b$ and $\delta^{\prime}=b_{1} \beta^{-1 / 2}-b$, and $b=\min \left\{\hat{b}_{1} \beta_{n}^{-1 / 2}, b_{1} \beta^{-1 / 2}\right\}$. Define the random vectors $\overline{\mathbf{L}}^{(2)}$ and $\overline{\mathbf{L}}^{(3)}$ in the same way as $\mathbf{L}^{(2)}$ and $\mathbf{L}^{(3)}$ above, but with $\xi_{2 i}$ and $\xi_{3 i}$ replaced by 
$\bar{\xi}_{2 i}=\xi_{i}+\Delta_{i}^{\prime}$ and $\bar{\xi}_{3 i}=\xi_{i}+\Delta_{i}^{\prime \prime}$ respectively. We note that given $Y_{1}, Y_{2}, X_{1}$ the random vector $\overline{\mathbf{L}}^{(2)}$ has the same conditional distribution as $\mathbf{L}^{(2)}$, and $\overline{\mathbf{L}}^{(3)}$ has the same conditional distribution as $\mathbf{L}^{(3)}$. Hence in order to prove 54 for $h=2$ it suffices to show that for any realized values $Y_{1}, Y_{2}, X_{1}$ we have

$$
\mathbf{P}^{\star}\left(\left|\bar{L}_{k}^{(2)}-\bar{L}_{k}^{(3)}\right| \geq 1\right)=o(1), \quad k=0,1,2 .
$$

Here $\bar{L}_{k}^{(i)}$ denotes the $k$-th coordinate of the vector $\overline{\mathbf{L}}^{(i)}$. We derive 64 from the bounds $\tilde{\mathbf{E}}\left|\bar{L}_{k}^{(2)}-\bar{L}_{k}^{(3)}\right|=o_{P^{\star}}(1)$ using 31 . To prove these bounds we calculate

$$
\begin{aligned}
\tilde{\mathbf{E}}\left|\bar{L}_{0}^{(2)}-\bar{L}_{0}^{(3)}\right|=\left(\delta^{\prime}+\delta^{\prime \prime}\right) X_{1}, \\
\tilde{\mathbf{E}}\left|\bar{L}_{k}^{(2)}-\bar{L}_{k}^{(3)}\right|=\sum_{2 \leq i \leq m} \tilde{\mathbf{E}} \eta_{k i}\left(\delta^{\prime}+\delta^{\prime \prime}\right) X_{i}=\beta_{n}^{1 / 2} Y_{k} \hat{a}_{2}\left(\delta^{\prime}+\delta^{\prime \prime}\right), \quad k=1,2
\end{aligned}
$$

and use the fact that $\delta^{\prime}+\delta^{\prime \prime}=\left|\hat{b}_{1} \beta_{n}^{-1 / 2}-b_{1} \beta^{-1 / 2}\right|=o_{P}(1)$. Indeed, we have $\beta_{n} \rightarrow \beta$ and, by the law of large numbers, $\hat{b}_{1}-b_{1}=o_{P}(1)$.

Let us prove 54 for $h=3$. We note that $L_{0}^{(3)}$ has the same distribution as $\Lambda_{0}$. Furthermore, given $Y_{1}, Y_{2}, X_{1}$, the random variable $L_{0}^{(3)}$ is conditionally independent of $\left(L_{1}^{(3)}, L_{2}^{(3)}\right)$. Hence, it suffices to show that $\mathbf{P}^{*}\left(\left(L_{1}^{(3)}, L_{2}^{(3)}\right)=\left(r_{1}, r_{2}\right)\right)-\mathbf{P}^{*}\left(\left(L_{1}^{(4)}, L_{2}^{(4)}\right)=\left(r_{1}, r_{2}\right)\right)=o(1)$ for any integers $r_{1}, r_{2} \geq 0$. For this purpose we prove the convergence of the conditional characteristic functions

$$
\mathbf{E}^{\star} e^{\mathbf{i} t L_{1}^{(3)}+\mathbf{i} s L_{2}^{(3)}} \rightarrow \mathbf{E}^{\star} e^{\mathbf{i} t L_{1}^{(4)}+\mathbf{i} s L_{2}^{(4)}}, \quad \forall s, t \in(-\infty,+\infty) .
$$

Here $\mathbf{i}$ denotes the imaginary unit.

In the proof of 65 we exploit the compound Poisson structure of the distributions of $L_{k}^{(h)}$, $h=2,3, k=1,2$. Using the fact that, given $Y_{1}, Y_{2}, X_{1}$, the marginals of $\left(L_{1}^{(4)}, L_{2}^{(4)}\right)$ are conditionally independent and have compound Poisson distributions, we write the characteristics function in the form

$$
\mathbf{E}^{\star} e^{\mathbf{i} t L_{1}^{(4)}+\mathbf{i} s L_{2}^{(4)}}=\left(\mathbf{E}^{\star} e^{\mathbf{i} t L_{1}^{(4)}}\right) \cdot\left(\mathbf{E}^{\star} e^{\mathrm{i} s L_{2}^{(4)}}\right)=e^{\left(f_{\tau}(t)-1\right) \lambda_{1}} \cdot e^{\left(f_{\tau}(s)-1\right) \lambda_{2}} \cdot=: f_{1}(t) \cdot f_{2}(s) .
$$

Here $f_{\tau}(t)=\mathbf{E} e^{i t \tau}$ denotes the characteristic function of $\tau$. Similarly, using the fact that given $X, Y, \xi_{32}, \ldots, \xi_{3 m}$ the marginals of $\left(L_{1}^{(3)}, L_{2}^{(3)}\right)$ are conditionally independent and have compound Poisson distributions, we write the conditional characteristics function in the form

$$
\hat{\mathbf{E}} e^{\mathbf{i} t L_{1}^{(3)}+\mathbf{i} s L_{2}^{(3)}}=\left(\hat{\mathbf{E}} e^{\mathbf{i} t L_{1}^{(3)}}\right) \cdot\left(\hat{\mathbf{E}} e^{\mathbf{i} s L_{2}^{(3)}}\right)=e^{\left(\hat{f}_{\tau}(t)-1\right) \hat{\lambda}_{1}} \cdot e^{\left(\hat{f}_{\tau}(s)-1\right) \hat{\lambda}_{2}}=: g_{1}(t) \cdot g_{2}(s) .
$$

Here $\hat{\mathbf{E}}$ denotes the conditional expectation given $X, Y, \xi_{32}, \ldots, \xi_{3 m}$. Furthermore, we denote $\hat{\lambda}_{k}=\sum_{2 \leq j \leq m} \lambda_{j k}$ and

$$
\hat{f}_{\tau}(t)=\sum_{r \geq 0} e^{i t r} \hat{p}_{r}, \quad \text { where } \quad \hat{p}_{r}=\hat{\lambda}_{1}^{-1} \sum_{2 \leq j \leq m} \lambda_{j 1} \mathbb{I}_{\left\{\xi_{3 j}=r\right\}} .
$$

We observe that $\hat{\lambda}_{k}=Y_{k} \hat{a}_{1} \sqrt{\beta_{n}}$ and each ratio $\lambda_{j k} / \hat{\lambda}_{k}=X_{j} / \hat{a}_{1}$ does not depend on $k=1,2$.

Finally, using (66), (67) we write

$$
\mathbf{E}^{\star} e^{\mathbf{i} t L_{1}^{(3)}+\mathbf{i} s L_{2}^{(3)}}-\mathbf{E}^{\star} e^{\mathbf{i} t L_{1}^{(4)}+\mathbf{i} s L_{2}^{(4)}}=\mathbf{E}^{\star}\left(g_{1}(t)-f_{1}(t)\right) g_{2}(s)+\mathbf{E}^{\star}\left(g_{2}(s)-f_{2}(s)\right) f_{1}(t)
$$

and invoke the bounds $\mathbf{E}^{\star}\left(g_{1}(t)-f_{1}(t)\right) g_{2}(s)=o(1)$ and $\mathbf{E}^{\star}\left(g_{2}(s)-f_{2}(s)\right) f_{1}(t)=o(1)$, which are obtained in the same way as relation (22) in [4]. We note that the proof of (22) in [4] uses the moment conditions $\mathbf{E} X_{1}^{2}<\infty, \mathbf{E} Y_{1}<\infty$ that are assumed to hold in the statement of our Theorem 2, 
Lemma 3. Assume that conditions of part (ii) of Theorem 2 are satisfied. Then for any realized values $Y_{1}, Y_{2}, X_{1}$ and any integers $k_{1}, k_{2} \geq 0$ we have

$$
\mathbf{P}^{\star}\left(L_{1}=k_{1}, L_{2}=k_{2}\right)-\mathbf{P}^{\star}\left(\mathcal{U}_{k_{1}, k_{2}}^{* * *}\right)=o(1) .
$$

Proof of Lemma 3. Before the proof we introduce some notation. Let $\varepsilon>0$. For $2 \leq i \leq m$ denote

$$
\mathbb{I}_{i}^{\prime}=\mathbb{I}_{i}^{\prime}(\varepsilon)=\mathbb{I}_{\left\{X_{i} \beta_{n}^{-1 / 2} b_{1}<\varepsilon\right\}}, \quad \gamma_{i}=X_{i} \beta_{n}^{-1 / 2} b_{1} \mathbb{I}_{i}^{\prime} \quad \text { and } \quad \theta_{k}=\sum_{2 \leq i \leq m} \lambda_{i k} \gamma_{i}, \quad k=1,2 .
$$

Given $X, Y$, let $\tilde{\mathbb{I}}_{2}, \ldots, \tilde{\mathbb{I}}_{m}$ be independent Bernoulli random variables with success probabilities

$$
\tilde{\mathbf{P}}\left(\tilde{\mathbb{I}}_{i}=1\right)=1-\tilde{\mathbf{P}}\left(\tilde{\mathbb{I}}_{i}=0\right)=\gamma_{i},
$$

and let $\bar{\xi}_{h i}, 2 \leq i \leq m, h=1,2$, be independent Poisson random variables with mean values

$$
\tilde{\mathbf{E}} \bar{\xi}_{1 i}=\sum_{3 \leq j \leq n} \lambda_{i j}, \quad \tilde{\mathbf{E}} \bar{\xi}_{2 i}=\frac{b_{1}}{\sqrt{\beta_{n}}} X_{i} .
$$

We assume that given $X, Y$ the sequences $\left\{\mathbb{I}_{i}\right\}_{i=2}^{m},\left\{\tilde{\mathbb{I}}_{i}\right\}_{i=2}^{m}$, and $\left\{\bar{\xi}_{h i}\right\}_{i=2}^{m}, h=1,2$ are independent. Next, we introduce random vectors $\overline{\mathbf{L}}^{(h)}=\left(\bar{L}_{1}^{(h)}, \bar{L}_{2}^{(h)}\right), 0 \leq h \leq 6$. Denote

$$
\overline{\mathbf{L}}^{(0)}=\left(\bar{L}_{1}^{(0)}, \bar{L}_{2}^{(0)}\right):=\left(L_{1}, L_{2}\right), \quad \overline{\mathbf{L}}^{(6)}=\left(\bar{L}_{1}^{(6)}, \bar{L}_{2}^{(6)}\right):=\left(\Lambda_{3}, \Lambda_{4}\right) .
$$

For $k=1,2$ and $h=1,2$ denote

$$
\bar{L}_{k}^{(h)}=\sum_{2 \leq i \leq m} \mathbb{I}_{i k} \bar{\xi}_{h i}, \quad \bar{L}_{k}^{(3)}=\sum_{2 \leq i \leq m} \mathbb{I}_{i k} \mathbb{I}_{i}^{\prime} \bar{\xi}_{2 i}, \quad \bar{L}_{k}^{(4)}=\sum_{2 \leq i \leq m} \mathbb{I}_{i k} \tilde{\mathbb{I}}_{i} .
$$

Furthermore, given $X, Y$, let $\bar{L}_{1}^{(5)}$ and $\bar{L}_{2}^{(5)}$ be independent Poisson random variables with mean values $\tilde{\mathbf{E}} \bar{L}_{k}^{(5)}=\theta_{k}$.

We shall show below that for any integers $r_{1}, r_{2} \geq 0$ we have

$$
\begin{aligned}
& d_{T V}^{\star}\left(\mathbf{L}^{(0)}, \mathbf{L}^{(1)}\right)=o(1), \\
& \mathbf{P}^{\star}\left(\overline{\mathbf{L}}^{(1)}=\left(r_{1}, r_{2}\right)\right)-\mathbf{P}^{\star}\left(\overline{\mathbf{L}}^{(2)}=\left(r_{1}, r_{2}\right)\right)=o(1), \\
& \mathbf{E}^{\star}\left|L_{k}^{(2)}-L_{k}^{(3)}\right|=o(1), \quad k=1,2, \\
& d_{T V}^{\star}\left(\mathbf{L}^{(3)}, \mathbf{L}^{(4)}\right) \leq \varepsilon\left(Y_{1}+Y_{2}\right) a_{2} b_{1}, \\
& \left|\mathbf{P}^{\star}\left(\overline{\mathbf{L}}^{(4)}=\left(r_{1}, r_{2}\right)\right)-\mathbf{P}^{\star}\left(\overline{\mathbf{L}}^{(6)}=\left(r_{1}, r_{2}\right)\right)\right| \leq \varepsilon\left(Y_{1}+Y_{2}\right) a_{2} b_{1}+o(1) .
\end{aligned}
$$

Since $\varepsilon>0$ can be chosen arbitrarily small, relations (69), (70), 711), (72), and (73) imply 68).

Let us prove (69). Introduce random variables

$$
\bar{L}_{k}^{\{t\}}=\sum_{2 \leq i \leq t} \mathbb{I}_{i k} u_{i}+\sum_{t+1 \leq i \leq m} \mathbb{I}_{i k} \bar{\xi}_{1 i}, \quad t=1, \ldots, m, \quad k=1,2 .
$$

Note that $\bar{L}_{k}^{\{1\}}=\bar{L}_{k}^{(1)}$ and $\bar{L}_{k}^{\{m\}}=\bar{L}_{k}^{(0)}$. We apply triangle inequality

$$
\tilde{d}_{T V}\left(\left(\bar{L}_{1}^{\{m\}}, \bar{L}_{2}^{\{m\}}\right),\left(\bar{L}_{1}^{\{1\}}, \bar{L}_{2}^{\{1\}}\right)\right) \leq \sum_{t=2}^{m} \tilde{d}_{T V}\left(\left(\bar{L}_{1}^{\{t-1\}}, \bar{L}_{2}^{\{t-1\}}\right),\left(\bar{L}_{1}^{\{t\}}, \bar{L}_{2}^{\{t\}}\right)\right)
$$


and estimate each summand as follows

$$
\begin{aligned}
\tilde{d}_{T V}\left(\left(\bar{L}_{1}^{\{t-1\}}, \bar{L}_{2}^{\{t-1\}}\right),\left(\bar{L}_{1}^{\{t\}}, \bar{L}_{2}^{\{t\}}\right)\right) & \leq \tilde{d}_{T V}\left(\left(\mathbb{I}_{t 1} u_{t}, \mathbb{I}_{t 2} u_{t}\right),\left(\mathbb{I}_{t 1} \bar{\xi}_{1 t}, \mathbb{I}_{t 2} \bar{\xi}_{1 t}\right)\right) \\
& \leq \tilde{\mathbf{P}}\left(\left(\mathbb{I}_{t 1}, \mathbb{I}_{t 2}\right) \neq(0,0)\right) \tilde{d}_{T V}\left(u_{t}, \bar{\xi}_{1 t}\right) \\
& \leq\left(\lambda_{t 1}+\lambda_{t 2}\right) \beta_{n}^{-1 / 2} X_{t}\left(\hat{b}_{2} n^{-1}\right)^{1 / 2} .
\end{aligned}
$$

In 75 we use the fact that $\bar{L}_{k}^{\{t-1\}}$ and $\bar{L}_{k}^{\{t\}}$ only differ in the $t$-th summand. In 76 we first estimate the total variation distance between conditional distributions of $\left(\mathbb{I}_{t 1} u_{t}, \mathbb{I}_{t 2} u_{t}\right)$ and $\left(\mathbb{I}_{t 1} \bar{\xi}_{1 t}, \mathbb{I}_{t 2} \bar{\xi}_{1 t}\right)$ given $\mathbb{I}_{t 1}, \mathbb{I}_{t 2}, X, Y$ from above by $\mathbb{I}_{\left\{\left(\eta_{t 1}, \eta_{t 2}\right) \neq(0,0)\right\}} \tilde{d}_{T V}\left(u_{t}, \bar{\xi}_{1 t}\right)$ and then take the expected value $\tilde{\mathbf{E}}$. In $\sqrt{77}$ we estimate $\tilde{\mathbf{P}}\left(\left(\mathbb{I}_{t 1}, \mathbb{I}_{t 2}\right) \neq(0,0)\right) \leq \lambda_{t 1}+\lambda_{t 2}$ and invoke the inequality

$$
\tilde{d}_{T V}\left(u_{t}, \bar{\xi}_{1 t}\right) \leq \min \left\{1, \beta_{n}^{-1} X_{t}^{2} \hat{b}_{2} n^{-1}\right\} \leq\left(\beta_{n}^{-1} X_{t}^{2} \hat{b}_{2} n^{-1}\right)^{1 / 2},
$$

cf. (57) above.

Next, using the identities $\bar{L}_{k}^{\{1\}}=\bar{L}_{k}^{(1)}$ and $\bar{L}_{k}^{\{m\}}=\bar{L}_{k}^{(0)}$ we obtain from 74 , 77 that

$$
\tilde{d}_{T V}\left(\overline{\mathbf{L}}^{(0)}, \overline{\mathbf{L}}^{(1)}\right) \leq \sum_{t=2}^{m}\left(\lambda_{t 1}+\lambda_{t 2}\right) \beta_{n}^{-1 / 2} X_{t}\left(\hat{b}_{2} n^{-1}\right)^{1 / 2}=\left(Y_{1}+Y_{2}\right) \hat{a}_{2}\left(\hat{b}_{2} / n\right)^{1 / 2} .
$$

Finally, since $\mathbf{E} X_{1}^{2}<\infty$ and $\mathbf{E} Y_{1}<\infty$ imply $\hat{a}_{2}=O_{P}(1)$ and $\hat{b}_{2} / n=o_{P}(1)$, we conclude that $\tilde{d}_{T V}\left(\overline{\mathbf{L}}^{(0)}, \overline{\mathbf{L}}^{(1)}\right)=o_{P^{\star}}(1)$. Hence,

$$
d_{T V}^{\star}\left(\overline{\mathbf{L}}^{(0)}, \overline{\mathbf{L}}^{(1)}\right)=\mathbf{E}^{\star} \tilde{d}_{T V}\left(\overline{\mathbf{L}}^{(0)}, \overline{\mathbf{L}}^{(1)}\right)=\mathbf{E}^{\star} \min \left\{1, \tilde{d}_{T V}\left(\overline{\mathbf{L}}^{(0)}, \overline{\mathbf{L}}^{(1)}\right)\right\}=o(1) .
$$

Let us prove 70 . We proceed as in the proof of 54 for $h=2$ above. Denote $b=\min \left\{\hat{b}_{1}, b_{1}\right\}$ and $\delta^{\prime}=\hat{b}_{1}-b, \delta^{\prime \prime}=b_{1}-b$. Given $X, Y$, let $\hat{\xi}_{i}, \Delta_{i}^{\prime}, \hat{\Delta}_{i}^{\prime \prime}, 2 \leq i \leq m$, be independent Poisson random variables, which are independent of $\mathbb{I}_{2}, \ldots, \mathbb{I}_{m}$, and having mean values

$$
\tilde{\mathbf{E}} \hat{\xi}_{i}=\beta_{n}^{-1 / 2} X_{i} b, \quad \tilde{\mathbf{E}} \hat{\Delta}_{i}^{\prime}=\beta_{n}^{-1 / 2} X_{i} \delta^{\prime}, \quad \tilde{\mathbf{E}} \hat{\Delta}_{i}^{\prime \prime}=\beta_{n}^{-1 / 2} X_{i} \delta^{\prime \prime} .
$$

Denote

$$
\hat{L}_{k}^{(1)}=\sum_{2 \leq i \leq m} \mathbb{I}_{i k}\left(\hat{\xi}_{i}+\hat{\Delta}_{i}^{\prime}\right), \quad \hat{L}_{k}^{(2)}=\sum_{2 \leq i \leq m} \mathbb{I}_{i k}\left(\hat{\xi}_{i}+\hat{\Delta}_{i}^{\prime \prime}\right), \quad k=1,2 .
$$

We note that $\hat{\mathbf{L}}^{(h)}:=\left(\hat{L}_{1}^{(h)}, \hat{L}_{2}^{(h)}\right)$ has the same distribution as $\overline{\mathbf{L}}^{(h)}, h=1,2$. Hence it suffices to show 70 for $\hat{\mathbf{L}}^{(1)}, \hat{\mathbf{L}}^{(2)}$. Proceeding as in the proof of 54 for $h=2$ above, we reduce the problem to showing that

$$
\tilde{\mathbf{E}}\left|\hat{L}_{k}^{(1)}-\hat{L}_{k}^{(2)}\right|=\sum_{2 \leq i \leq m} \lambda_{i k} \beta_{n}^{-1}\left(\delta^{\prime}+\delta^{\prime \prime}\right)=Y_{k} \hat{a}_{2}\left|\hat{b}_{1}-b_{1}\right|=o(1), \quad k=1,2 .
$$

Let us prove (71). We have, for $k=1,2$,

$$
\tilde{\mathbf{E}}\left|L_{k}^{(2)}-L_{k}^{(3)}\right|=\sum_{2 \leq i \leq m}\left(1-\mathbb{I}_{i}^{\prime}\right)\left(\tilde{\mathbf{E}} \mathbb{I}_{i k}\right)\left(\tilde{\mathbf{E}} \bar{\xi}_{2 i}\right) \leq Y_{k} b_{1} m^{-1} \sum_{2 \leq i \leq m}\left(1-\mathbb{I}_{i}^{\prime}\right) X_{i}^{2}
$$

Taking $\mathbf{E}^{\star}$ expected value we obtain

$$
\mathbf{E}^{\star}\left|L_{k}^{(2)}-L_{k}^{(3)}\right|=\mathbf{E}^{\star}\left(\tilde{\mathbf{E}}\left|L_{k}^{(2)}-L_{k}^{(3)}\right|\right) \leq Y_{k} b_{1} \mathbf{E} X_{2}^{2} \mathbb{I}_{\left\{X_{2} \beta_{n}^{-1 / 2} b_{1} \geq \varepsilon\right\}}=o(1) .
$$


We note that the expectation on right tends to zero since $\beta_{n} \rightarrow+\infty$.

Let us prove $(72)$. We proceed as in the proof of $(69)$ for $h=0$ above. We have

$$
\left.\tilde{d}_{T V}\left(\mathbf{L}^{(3)}, \mathbf{L}^{(4)}\right) \leq \sum_{2 \leq i \leq m} \mathbb{I}_{i}^{\prime} \tilde{\mathbf{P}}\left(\mathbb{I}_{i 1}, \mathbb{I}_{i 2}\right) \neq(0,0)\right) \tilde{d}_{T V}\left(\bar{\xi}_{2 i}, \tilde{\mathbb{I}}_{i}\right) .
$$

Next, we estimate $\mathbb{I}_{i}^{\prime} \tilde{d}_{T V}\left(\bar{\xi}_{2 i}, \tilde{\mathbb{I}}_{i}\right) \leq \gamma_{i}^{2}$, by Le Cam's inequality $[51$, and invoke the inequality $\left.\tilde{\mathbf{P}}\left(\mathbb{I}_{i 1}, \mathbb{I}_{i 2}\right) \neq(0,0)\right) \leq \lambda_{i 1}+\lambda_{i 2}$. We obtain

$$
\tilde{d}_{T V}\left(\mathbf{L}^{(3)}, \mathbf{L}^{(4)}\right) \leq \sum_{2 \leq i \leq m} \mathbb{I}_{i}^{\prime}\left(\lambda_{i 1}+\lambda_{i 2}\right) \gamma_{i}^{2} \leq \varepsilon \sum_{2 \leq i \leq m} \mathbb{I}_{i}^{\prime}\left(\lambda_{i 1}+\lambda_{i 2}\right) \gamma_{i} \leq \varepsilon\left(Y_{1}+Y_{2}\right) b_{1} \hat{a}_{2} .
$$

Here we used inequality $\gamma_{i}^{2} \leq \varepsilon \gamma_{i}$. It follows now that

$$
d_{T V}^{\star}\left(\mathbf{L}^{(3)}, \mathbf{L}^{(4)}\right) \leq \mathbf{E}^{\star} \tilde{d}_{T V}\left(\mathbf{L}^{(3)}, \mathbf{L}^{(4)}\right) \leq \varepsilon\left(Y_{1}+Y_{2}\right) b_{1} a_{2} .
$$

Let us prove 73 . For $A \subset W$ denote $\tilde{S}(A)=\sum_{w_{i} \in A} \tilde{\mathbb{I}}_{i}$. For $k=1,2$ we write $\bar{L}_{k}^{(4)}=\tilde{S}\left(\mathbf{A}_{k}\right)$, where $\mathbf{A}_{k}$ denote the set attributes from $W \backslash\left\{w_{1}\right\}$ that are prescribed to $v_{k}$, i.e., $\mathbf{A}_{k}=\left\{w_{i} \in\right.$ $\left.W \backslash\left\{w_{1}\right\}: w_{i} \rightarrow v_{k}\right\}$. Given $r_{1}, r_{2} \geq 0$ introduce events $\mathcal{D}_{k}=\left\{\tilde{S}\left(\mathbf{A}_{k}\right)=r_{k}\right\}, k=1,2$, and $\mathcal{D}=\left\{\left|\mathbf{A}_{1} \cap \mathbf{A}_{2}\right| \geq 1\right\}$.

We first show that

$$
\mathbf{P}^{\star}\left(\bar{L}_{1}^{(4)}=r_{1}, \bar{L}_{2}^{(4)}=r_{2}\right)=\mathbf{E}^{\star}\left(\tilde{\mathbf{P}}\left(\bar{L}_{1}^{(4)}=r_{1}\right) \tilde{\mathbf{P}}\left(\bar{L}_{2}^{(4)}=r_{2}\right)\right)+o(1) .
$$

It is convenient to write $(79)$ in the form

$$
\mathbf{P}^{\star}\left(\mathcal{D}_{1} \cap \mathcal{D}_{2}\right)=\mathbf{E}^{\star}\left(\tilde{\mathbf{P}}\left(\mathcal{D}_{1}\right) \tilde{\mathbf{P}}\left(\mathcal{D}_{2}\right)\right)+o(1) .
$$

Now, we observe that given $X, Y$, and $\mathbf{A}_{1}, \mathbf{A}_{2}$, satisfying $\mathbf{A}_{1} \cap \mathbf{A}_{2}=\emptyset$, the random variables $\tilde{S}\left(\mathbf{A}_{1}\right)$ and $\tilde{S}\left(\mathbf{A}_{2}\right)$ are independent. Hence for $\mathbf{A}_{1} \cap \mathbf{A}_{2}=\emptyset$ we have

$$
\tilde{\mathbf{P}}\left(\mathcal{D}_{1} \cap \mathcal{D}_{2} \mid \mathbf{A}_{1}, \mathbf{A}_{2}\right)=\tilde{\mathbf{P}}\left(\mathcal{D}_{1} \mid \mathbf{A}_{1}\right) \tilde{\mathbf{P}}\left(\mathcal{D}_{2} \mid \mathbf{A}_{2}\right) .
$$

This identity implies

$$
\tilde{\mathbf{P}}\left(\mathcal{D}_{1} \cap \mathcal{D}_{2} \cap \overline{\mathcal{D}}\right)=\tilde{\mathbf{E}}\left(\tilde{\mathbf{P}}\left(\mathcal{D}_{1} \cap \mathcal{D}_{2} \mid \mathbf{A}_{1}, \mathbf{A}_{2}\right) \overline{\mathbb{I}}_{\mathcal{D}}\right)=\tilde{\mathbf{E}}\left(\tilde{\mathbf{P}}\left(\mathcal{D}_{1} \mid \mathbf{A}_{1}\right) \tilde{\mathbf{P}}\left(\mathcal{D}_{2} \mid \mathbf{A}_{2}\right) \overline{\mathbb{I}}_{\mathcal{D}}\right) .
$$

Next, combining inequalities

$$
\begin{aligned}
& 0 \leq \tilde{\mathbf{P}}\left(\mathcal{D}_{1} \cap \mathcal{D}_{2}\right)-\tilde{\mathbf{P}}\left(\mathcal{D}_{1} \cap \mathcal{D}_{2} \cap \overline{\mathcal{D}}\right) \leq \tilde{\mathbf{P}}(\mathcal{D}) \\
& 0 \leq \tilde{\mathbf{E}}\left(\tilde{\mathbf{P}}\left(\mathcal{D}_{1} \mid \mathbf{A}_{1}\right) \tilde{\mathbf{P}}\left(\mathcal{D}_{2} \mid \mathbf{A}_{2}\right)\right)-\tilde{\mathbf{E}}\left(\tilde{\mathbf{P}}\left(\mathcal{D}_{1} \mid \mathbf{A}_{1}\right) \tilde{\mathbf{P}}\left(\mathcal{D}_{2} \mid \mathbf{A}_{2}\right) \overline{\mathbb{I}}_{\mathcal{D}}\right) \leq \tilde{\mathbf{P}}(\mathcal{D})
\end{aligned}
$$

with 80 and using the identity (which holds, since given $X, Y$, the random sets $\mathbf{A}_{1}$ and $\mathbf{A}_{2}$ are independent)

$$
\tilde{\mathbf{E}}\left(\tilde{\mathbf{P}}\left(\mathcal{D}_{1} \mid \mathbf{A}_{1}\right) \tilde{\mathbf{P}}\left(\mathcal{D}_{2} \mid \mathbf{A}_{2}\right)\right)=\left(\tilde{\mathbf{E}} \tilde{\mathbf{P}}\left(\mathcal{D}_{1} \mid \mathbf{A}_{1}\right)\right)\left(\tilde{\mathbf{E}} \tilde{\mathbf{P}}\left(\mathcal{D}_{2} \mid \mathbf{A}_{2}\right)\right)=\tilde{\mathbf{P}}\left(\mathcal{D}_{1}\right) \tilde{\mathbf{P}}\left(\mathcal{D}_{2}\right)
$$

we obtain that

$$
\left|\mathbf{P}^{\star}\left(\mathcal{D}_{1} \cap \mathcal{D}_{2}\right)-\mathbf{E}^{\star}\left(\tilde{\mathbf{P}}\left(\mathcal{D}_{1}\right) \tilde{\mathbf{P}}\left(\mathcal{D}_{2}\right)\right)\right| \leq \mathbf{E}^{\star} \tilde{\mathbf{P}}(\mathcal{D}) .
$$

It remains to show that $\mathbf{E}^{\star} \tilde{\mathbf{P}}(\mathcal{D})=o(1)$. To this aim we apply Markov's inequality

$$
\tilde{\mathbf{P}}(\mathcal{D}) \leq \sum_{2 \leq i \leq m} \tilde{\mathbf{E}} \mathbb{I}_{i 1} \mathbb{I}_{i 2} \leq \sum_{2 \leq i \leq m} \mathbf{E} \lambda_{i 1} \lambda_{i 2}=n^{-1} Y_{1} Y_{2} \hat{a}_{2}
$$


and obtain $\mathbf{E}^{\star}(\tilde{\mathbf{P}}(\mathcal{D})) \leq n^{-1} Y_{1} Y_{2} a_{2}=o(1)$ thus completing the proof of 79 .

We secondly show that

$$
\mathbf{E}^{\star}\left|\tilde{\mathbf{P}}\left(\bar{L}_{1}^{(4)}=r_{1}\right) \tilde{\mathbf{P}}\left(\bar{L}_{2}^{(4)}=r_{2}\right)-\tilde{\mathbf{P}}\left(\bar{L}_{1}^{(5)}=r_{1}\right) \tilde{\mathbf{P}}\left(\bar{L}_{2}^{(5)}=r_{2}\right)\right| \leq \varepsilon\left(Y_{1}+Y_{2}\right) b_{1} a_{2} .
$$

Denote, for short, the integrand in 82$)$ by $\left|q_{1} q_{2}-h_{1} h_{2}\right|$. Since $0 \leq q_{1}, q_{2}, h_{1}, h_{2} \leq 1$, we have $\left|q_{1} q_{2}-h_{1} h_{2}\right| \leq\left|q_{1}-h_{1}\right|+\left|q_{2}-h_{2}\right|$. We apply Le Cam's inequality (51) and obtain

$$
\left|q_{k}-h_{k}\right| \leq \sum_{2 \leq i \leq m} \mathbb{I}_{i}^{\prime} \lambda_{i k} \gamma_{i}^{2} \leq \varepsilon \sum_{2 \leq i \leq m} \mathbb{I}_{i}^{\prime} \lambda_{i k} \gamma_{i} \leq \varepsilon Y_{1} b_{1} \hat{a}_{2}, \quad k=1,2
$$

Here we estimate $\gamma_{i}^{2} \leq \varepsilon \gamma_{i}$. Clearly, inequality $\mathbf{E}^{\star}\left|q_{k}-h_{k}\right| \leq \varepsilon Y_{k} b_{1} a_{2}$ imply (82).

Finally, we show that

$$
\mathbf{E}^{\star}\left(\tilde{\mathbf{P}}\left(\bar{L}_{1}^{(5)}=r_{1}\right) \tilde{\mathbf{P}}\left(\bar{L}_{2}^{(5)}=r_{2}\right)\right) \rightarrow \mathbf{P}^{\star}\left(\Lambda_{3}=r_{1}\right) \mathbf{P}^{\star}\left(\Lambda_{4}=r_{2}\right) .
$$

Here we will use the fact that the almost sure convergence $\hat{a}_{2} \rightarrow a_{2}$ implies the convergence in probability

$$
\left(\theta_{1}, \theta_{2}\right) \stackrel{P}{\rightarrow}\left(\lambda_{3}, \lambda_{4}\right) .
$$

Since for $s, t \geq 0$ the function $(s, t) \rightarrow e^{-t-s} s^{r_{1}} t^{r_{2}} /\left(r_{1} ! r_{2} !\right)$ is continuous and bounded, (84) implies the convergence of expected values

$$
\mathbf{E}^{\star} \frac{e^{-\theta_{1}} e^{-\theta_{2}} \theta_{1}^{r_{1}} \theta_{2}^{r_{2}}}{r_{1} ! r_{2} !} \rightarrow \mathbf{E}^{\star} \frac{e^{-\lambda_{3}} e^{-\lambda_{4}} \lambda_{3}^{r_{1}} \lambda_{4}^{r_{2}}}{r_{1} ! r_{2} !}=\frac{e^{-\lambda_{3}} e^{-\lambda_{4}} \lambda_{3}^{r_{1}} \lambda_{4}^{r_{2}}}{r_{1} ! r_{2} !} .
$$

We observe that the quantities on the left (right) sides of 83 and 85 are the same. Hence (85) implies (83).

It remains to prove 84. Denote $\hat{\lambda}_{k+2}=Y_{k} b_{1} \hat{a}_{2}, k=1,2$. We have for any $\delta>0$

$$
\mathbf{P}^{\star}\left(\left|\theta_{k}-\hat{\lambda}_{2+k}\right| \geq \delta\right) \leq \delta^{-1} \mathbf{E}^{\star}\left|\theta_{k}-\hat{\lambda}_{2+k}\right| \leq \delta^{-1} Y_{k} b_{1} \mathbf{E} X_{2}^{2}\left(1-\mathbb{I}_{2}^{\prime}\right)=o(1) .
$$

In the last step we used $\mathbf{E} X_{2}^{2}\left(1-\mathbb{I}_{2}^{\prime}\right)=o(1)$. Now, 84 follows from 86 and the bounds $\hat{\lambda}_{k+2}-\lambda_{k+2}=o_{P}(1), k=1,2$, which are simple consequences of the fact that $\mathbf{E} X_{1}^{2}<\infty$ implies $\hat{a}_{2}-a_{2}=o_{P}(1)$.

\section{Appendix B}

Lemma 4. Let $c, \varkappa, h>0$. Let $Z, \Lambda_{Z}$ be non-negative random variables such that $\mathbf{P}\left(\Lambda_{Z}=r\right)=\mathbf{E}\left(e^{-Z} Z^{r} / r !\right), r=0,1, \ldots$

(i) The relation $\mathbf{P}(Z>t)=(c+o(1)) t^{-\varkappa}$ as $t \rightarrow+\infty$ implies

$$
\mathbf{P}\left(\Lambda_{Z}>t\right)=(c+o(1)) t^{-\varkappa} \quad \text { as } \quad t \rightarrow+\infty .
$$

(ii) If $Z \in \mathcal{P}_{c, \varkappa}$ then $\mathbf{P}\left(\Lambda_{Z}=r\right) \approx c r^{-\varkappa}$.

(iii) If $h Z$ is integer valued and satisfies $\mathbf{P}(h Z=r) \approx c(h / r)^{\varkappa}$ then $\mathbf{P}\left(\Lambda_{Z}=r\right) \approx c h r^{-\varkappa}$.

Proof of Lemma 4. Let us prove (i). We first collect auxiliary inequalities. For a Poisson random variable $\Lambda$ with mean $z>0$ and $0<s<z<t$ we have, see [15],

$$
\mathbf{P}(\Lambda \geq t) \leq e^{-z}(e z / t)^{t}, \quad \mathbf{P}(\Lambda \leq s) \leq e^{-z}(e z / s)^{s} .
$$


For $0<x<1$ and $y>1$ we have

$$
\ln (1-x) \leq-x-0.5 x^{2}, \quad \ln (1+x) \leq x-0.25 x^{2}, \quad \ln (1+y) \leq y \ln 2 .
$$

In order to prove 877 we split the probability $\mathbf{P}\left(\Lambda_{Z}>t\right)=P_{1}+P_{2}+P_{3}$, where

$$
\begin{aligned}
& P_{1}=\mathbf{P}\left(\Lambda_{Z}>t, Z<t_{1}\right), \quad P_{2}=\mathbf{P}\left(\Lambda_{Z}>t, Z \in\left[t_{1}, t_{2}\right]\right), \quad P_{3}=\mathbf{P}\left(\Lambda_{Z}>t, Z>t_{2}\right), \\
& t_{1}=t(1-\varepsilon), \quad t_{2}=t(1+\varepsilon), \quad \varepsilon=t^{-1 / 3},
\end{aligned}
$$

and show that $P_{2}=(c+o(1)) t^{-\varkappa}$ and $P_{k}=o\left(t^{-\varkappa}\right), k=1,2$.

We have

$$
P_{2} \leq \mathbf{P}\left(t_{1} \leq Z \leq t_{2}\right)=(c+o(1))\left(t_{1}^{-\varkappa}-t_{2}^{-\varkappa}\right)=o\left(t^{-\varkappa}\right) .
$$

Next, we estimate $P_{1}$. Given $z<t_{1}$ we denote $\bar{z}=1-z / t$. Using (88), (89) we obtain

$$
\mathbf{P}\left(\Lambda_{Z}>t \mid Z=z\right) \leq e^{-z}(e z / t)^{t}=e^{t-z+t \ln (1-\bar{z})} \leq e^{t-z-t \bar{z}-0.5 t \bar{z}^{2}}=e^{-0.5 t \bar{z}^{2}} \leq e^{-0.5 t \varepsilon^{2}} .
$$

Hence, $P_{1}=\mathbf{E}\left(\mathbf{P}\left(\Lambda_{Z}>t \mid Z\right) \mathbb{I}_{\left\{Z<t_{1}\right\}}\right) \leq e^{-0.5 \varepsilon^{2} t}=o\left(t^{-\varkappa}\right)$. In order to evaluate $P_{3}$ we observe that $\mathbf{P}\left(Z>t_{2}\right)=(c+o(1)) t^{-\varkappa}$ and write

$$
P_{3}=\mathbf{P}\left(Z>t_{2}\right)-\mathbf{P}\left(\Lambda_{Z} \leq t, Z>t_{2}\right) .
$$

Then we estimate

$$
\mathbf{P}\left(\Lambda_{Z} \leq t, Z>t_{2}\right)=\mathbf{E}\left(\mathbf{P}\left(\Lambda_{Z} \leq t \mid Z\right) \mathbb{I}_{\{Z>t\}}\right)=o\left(t^{-\varkappa}\right)
$$

using the inequality

$$
\mathbf{P}\left(\Lambda_{Z} \leq t \mid Z=z\right) \leq \max \left\{e^{-0.25 \varepsilon^{2} t}, e^{-t \ln (e / 2)}\right\}, \quad \forall z>t_{2} .
$$

It remains to prove 90$)$. We have, see (88),

$$
\mathbf{P}\left(\Lambda_{Z} \leq t \mid Z=z\right) \leq e^{-z}(e z / t)^{t}=e^{t-z+t \ln (1+y)} .
$$

Here $y=z t^{-1}-1$. For $0<y<1$ the quantity on the right is less than $e^{-0.25 \varepsilon^{2} t}$, by the second inequality of (89). For $y \geq 1$, by the third inequality of (89), the quantity on the right is less than $e^{-(z-t)(1-\ln 2)} \leq e^{-t \ln (e / 2)}$, since $z-t=t y \geq t$.

Let us prove (ii). We only consider the case of integer valued $Z$. For an absolute continuous $Z$ proof is the same. Denote $\varepsilon_{r}=r^{-1 / 2} \ln r$. We split

$$
\begin{aligned}
& \mathbf{P}\left(\Lambda_{Z}=r\right)=\mathbf{E}\left(e^{-Z} Z^{r} / r !\right)=J_{1}+J_{2}+J_{3}, \quad J_{k}=\mathbf{E}\left(\mathbb{I}_{\left\{Z \in Q_{k}\right\}} e^{-Z} Z^{r} / r !\right), \\
& Q_{1}=\left[0, r\left(1-\varepsilon_{r}\right)\right), \quad Q_{2}=\left[r\left(1-\varepsilon_{r}\right), r\left(1+\varepsilon_{r}\right)\right], \quad Q_{3}=\left(r\left(1+\varepsilon_{r}\right),+\infty\right),
\end{aligned}
$$

and show that

$$
J_{1}=o\left(r^{-\varkappa}\right), \quad J_{3}=o\left(r^{-\varkappa}\right), \quad J_{2}=(c+o(1)) r^{-\varkappa} \quad \text { as } \quad r \rightarrow+\infty .
$$

In the proof we use the following bounds related to the integral representation of Euler's Gamma function. Given interval $Q \subset[0,+\infty)$, denote

$$
I_{Q}=\int_{Q} \frac{e^{-x} x^{r}}{r !} d x, \quad S_{Q}=\sum_{j \in Q_{k}} \frac{e^{-j} j^{r}}{r !} .
$$


For large $r$ we have

$$
I_{Q_{1}} \leq r^{-0.3 \ln r}, \quad I_{Q_{3}} \leq r^{-0.3 \ln r}, \quad 1-2 r^{-0.3 \ln r} \leq I_{Q_{2}} \leq 1 .
$$

Relations (91) follow from 92 and the approximation $S_{Q_{k}}=(1+o(1)) I_{Q_{k}}, k=1,2,3$,

$$
\begin{aligned}
& J_{k}=\sum_{j \in Q_{k}} \frac{e^{-j} j^{r}}{r !} \mathbf{P}(Z=r) \leq S_{Q_{k}}=(1+o(1)) I_{Q_{k}}, \quad k=1,3, \\
& J_{2}=\sum_{j \in Q_{2}} \frac{e^{-j} j^{r}}{r !} \mathbf{P}(Z=r)=\frac{c+o(1)}{r^{\varkappa}} S_{Q_{2}}=\frac{c+o(1)}{r^{\varkappa}}(1+o(1)) I_{Q_{2}} .
\end{aligned}
$$

For reader's convenience we provide a proof of $(92)$. We note that the third relation of $(92)$ follows from the first two and the well known fact that $I_{[0,+\infty)}=1$. In the proof, for sufficiently small $\varepsilon>0$, we apply the inequalities, which follow by Taylor's expansion,

$$
e^{\varepsilon}(1-\varepsilon) \leq 1-\varepsilon^{2} / 3, \quad e^{-\varepsilon}(1+\varepsilon) \leq 1-\varepsilon^{2} / 3 .
$$

Since the function $x \rightarrow e^{-x} x^{r}$ increases on $Q_{1}$, we have $I_{Q_{1}} \leq z e^{-z} z^{r} / r$ !, where $z=r\left(1-\varepsilon_{r}\right)$ is the right end-point of $Q_{1}$. Using Stirling's formula we obtain for large $r$

$$
z e^{-z} z^{r} / r !=(1+o(1)) e^{r \varepsilon_{r}}\left(1-\varepsilon_{r}\right)^{r+1}(r / 2 \pi)^{1 / 2} \leq r^{1 / 2}\left(1-\varepsilon_{r}^{2} / 3\right)^{r} \leq r^{-0.3 \ln r} .
$$

In the last step we used $1-\varepsilon \leq e^{-\varepsilon}$. Furthermore, for $y=r\left(1+\varepsilon_{r}\right)$ we have decreases on $Q_{3}$ we have

$$
I_{Q_{3}} \leq \frac{e^{-y} y^{r}}{r !} \int_{y}^{+\infty} e^{-(x-y)}\left(1+\frac{x-y}{y}\right)^{r} d x .
$$

Using Stirling's formula we obtain

$$
e^{-y} y^{r} / r !=(1+o(1))\left(e^{-\varepsilon_{r}}\left(1+\varepsilon_{r}\right)\right)^{r}(2 \pi r)^{-1 / 2} \leq r^{-1 / 2}\left(1-\varepsilon_{r}^{2} / 3\right)^{r} \leq r^{-0.5-0.3 \ln r} .
$$

Combining this inequality with the following upper bound on the integral of 93

$$
\int_{0}^{+\infty} e^{-t}(1+t / y)^{r} d t \leq \int_{0}^{+\infty} e^{-t} e^{(r t / y)} d t=1+\varepsilon_{r}^{-1} \leq r^{1 / 2}
$$

we obtain 92 . Here we used the inequality $(1+t / y)^{r} \leq e^{(r t / y)}$.

We omit the proof of (iii) since it is similar to that of (ii).

Acknowledgement. The present study was motivated by a question of Konstantin Avratchenkov about the degree-degree distribution in a random intersection graph. A discussion with Konstantin Avratchenkov and Jerzy Jaworski about the influence of the clustering property on the degree-degree distribution was the starting point of this research.

\section{References}

[1] A. Aleškevičienè, R. Leipus and J. Šiaulys. Tail behavior of random sums under consistent variation with applications to the compound renewal risk model. Extremes, 11:261-279, 2008.

[2] K. Avratchenkov, N. M. Markovich, J.K. Sreedharan. Distribution and dependence of extremes in network sampling processes. Computational Social Networks, 2:12, 2015. 
[3] M. Bloznelis. Degree-degree distribution in a power law random intersection graph with clustering. In D. F. Gleich, J. Komjathy, N. Litvak, Eds., Algorithms and Models for the Web Graph - 12th International Workshop, WAW 2015, Lecture Notes in Computer Science, 9476: 42-53, 2015.

[4] M. Bloznelis, J. Damarackas. Degree distribution of an inhomogeneous random intersection graph. Electron. J. Combin., 20(3), \#P3, 2013.

[5] M. Bloznelis, E. Godehardt, J. Jaworski, V. Kurauskas, and K. Rybarczyk. Recent Progress in Complex Network Analysis - Properties of Random Intersection Graphs. In B. Lausen, S. Krolak-Schwerdt, M. Bhmer, Eds., Data Science, Learning by Latent Structures, and Knowledge Discovery, Springer, 79-88, 2015.

[6] M. Bloznelis, J. Jaworski, and V. Kurauskas. Assortativity and clustering coefficient of sparse random intersection graphs. Electronic journal of probability, 18, N-38, 2013.

[7] M. Bloznelis, V. Kurauskas. Clustering function: Another view on clustering coefficient. Journal of Complex Networks, 4: 61-86, 2016.

[8] M. Boguna, R. Pastor-Satorras, and A. Vespignani. Epidemic spreading in complex networks with degree correlations. Statistical Mechanics of Complex Networks. Lecture Notes in Physics, 625:127-147, 2003.

[9] S. Foss, D. Korshunov, and S. Zachary. An Introduction to Heavy-Tailed and Subexponential Distributions. 2nd Edition Springer, New York, 2013.

[10] E. Godehardt and J. Jaworski. Two models of random intersection graphs and their applications. Electronic Notes in Discrete Mathematics, 10:129-132, 2001.

[11] E. A. Grechnikov. The degree distribution and the number of edges between vertices of given degrees in the Buckley-Osthus model of a random web graph. Internet Mathematics, 8:257-287, 2012.

[12] R. van der Hofstad, N. Litvak. Degree-degree dependencies in random graphs with heavytailed degrees. Internet Mathematics,10: 287-334, 2014.

[13] S. Janson, T. Łuczak and A. Ruciński. Random Graphs. Wiley, New York, 2001.

[14] M. Karoński, E. R. Scheinerman, and K. B. Singer-Cohen. On random intersection graphs: The subgraph problem. Combinatorics, Probability and Computing, 8:131-159, 1999.

[15] M. Mitzenmacher, E. Upfal. Probability and Computing. Randomized Algorithms and Probabilistic Analysis. Cambridge University Press. 2005.

[16] L. Muchnik, S. Pei, L. C. Parra, S. D. S. Reis, J. S. Jr Andrade, S. Havlin, and H. A. Makse. Origins of power-law degree distribution in the heterogeneity of human activity in social networks. Scientific Reports, 3: 1783, 2013.

[17] M. E. J. Newman, S. H. Strogatz, and D. J. Watts, Random graphs with arbitrary degree distributions and their applications, Physical Review E 64 (2001) 026118.

[18] M.E.J. Newman. Assortative mixing in networks. Physical review letters, 89(20):208701, 2002 . 
[19] M. E.J. Newman. Mixing patterns in networks. Physical Review E, 67(2):026126, 2003.

[20] J. M. Steele. Le Cam's inequality and Poisson approximations. The American Mathematical Monthly, 101:48-54, 1994. 